This article was downloaded by: [University of Southampton]

On: 26 May 2010

Access details: Access Details: [subscription number 908420906]

Publisher Taylor \& Francis

Informa Ltd Registered in England and Wales Registered Number: 1072954 Registered office: Mortimer House, 3741 Mortimer Street, London W1T 3JH, UK

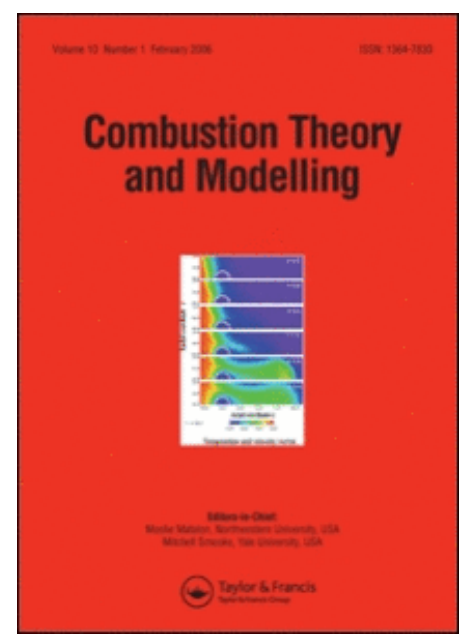

\title{
Combustion Theory and Modelling
}

Publication details, including instructions for authors and subscription information:

http://www.informaworld.com/smpp/title content=t713665226

\section{Stability of a spherical flame ball in a porous medium}

A. A. Shah ; R. W. Thatcher ; J. W. Dold ${ }^{\mathrm{a}}$

${ }^{a}$ Mathematics Department, UMIST, Manchester, UK

Online publication date: 10 November 2000

To cite this Article Shah, A. A. , Thatcher, R. W. and Dold, J. W.(2000) 'Stability of a spherical flame ball in a porous medium', Combustion Theory and Modelling, 4: 4, $511-534$

To link to this Article: DOI: $10.1088 / 1364-7830 / 4 / 4 / 308$

URL: http://dx.doi.org/10.1088/1364-7830/4/4/308

\section{PLEASE SCROLL DOWN FOR ARTICLE}

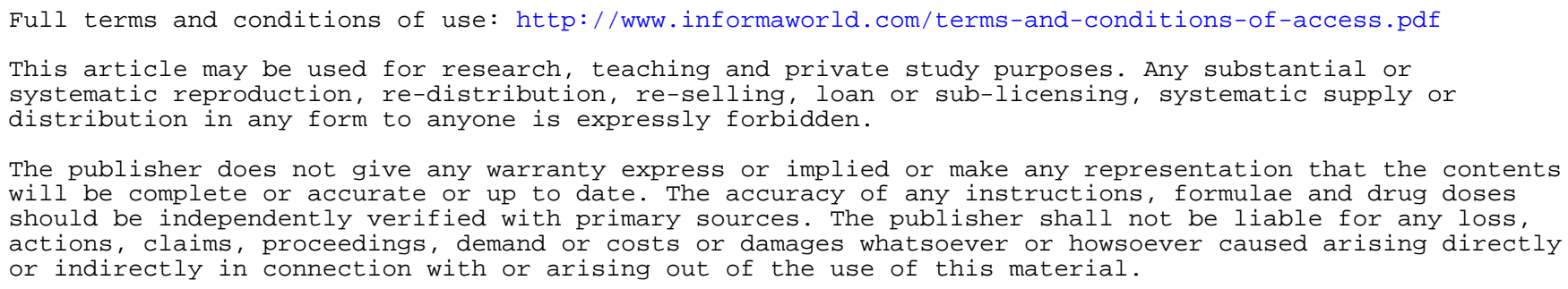




\title{
Stability of a spherical flame ball in a porous medium
}

\author{
A A Shah, R W Thatcher and J W Dold \\ Mathematics Department, UMIST, Manchester M60 1QD, UK \\ E-mail: John.Dold@umist.ac.uk
}

Received 24 July 2000, in final form 25 October 2000

\begin{abstract}
Gaseous flame balls and their stability to symmetric disturbances are studied numerically and asymptotically, for large activation temperature, within a porous medium that serves only to exchange heat with the gas. Heat losses to a distant ambient environment, affecting only the gas, are taken to be radiative in nature and are represented using two alternative models. One of these treats the heat loss as being constant in the burnt gases and linearizes the radiative law in the unburnt gas (as has been studied elsewhere without the presence of a solid). The other does not distinguish between burnt and unburnt gas and is a continuous dimensionless form of Stefan's law, having a linear part that dominates close to ambient temperatures and a fourth power that dominates at higher temperatures.

Numerical results are found to require unusually large activation temperatures in order to approach the asymptotic results. The latter involve two branches of solution, a smaller and a larger flame ball, provided heat losses are not too high. The two radiative heat loss models give completely analogous steady asymptotic solutions, to leading order, that are also unaffected by the presence of the solid which therefore only influences their stability. For moderate values of the dimensionless heat-transfer time between the solid and gas all flame balls are unstable for Lewis numbers greater than unity. At Lewis numbers less than unity, part of the branch of larger flame balls becomes stable, solutions with the continuous radiative law being stable over a narrower range of parameters. In both cases, for moderate heat-transfer times, the stable region is increased by the heat capacity of the solid in a way that amounts, simply, to decreasing an effective Lewis number for determining stability, just as if the heat-transfer time was zero.
\end{abstract}

\section{Introduction}

Flame balls are stationary spherically symmetric solutions of the equations describing premixed combustion [1]. Under adiabatic conditions they are unstable, but they are significant nevertheless, since a flame structure slightly bigger than the flame ball will grow, leading to propagating flame fronts, while a slightly smaller flame will collapse. They therefore have implications concerning the energy required for ignition [2-4].

Experimental observations at zero gravity [5] have found that spherical flame balls, in gases, can be produced as stable objects, if the Lewis number is low enough. It has also been shown, theoretically, that suitable non-adiabatic conditions can account for this stabilization $[6,7]$. Flame balls can be stabilized by uniform and constant heat losses only in the burnt gases [6] as well as by relatively weak linear heat losses that nevertheless are of leadingorder importance when temperatures are close to an ambient, far-field value [7]. Non-uniform velocity fields can also stabilize flame balls [8], while non-uniform enthalpy and boundary effects can induce some movement in a stable flame ball [9-12].

We extend these studies by introducing a model for gaseous flame balls within a porous, inert solid. The idea is to determine whether such a solid is likely to stabilize or destabilize 
a flame ball. The solid is treated, in this paper, as being immobile, non-conducting and nonradiating. Its only role is therefore to exchange heat with the gas, providing a form of heat reservoir for the gas at any point, representing a rather simple extension of existing models for flame balls. Such a reservoir would tend to resist temporal changes in the temperature of the gas and so it might be expected to offer a stabilizing influence which we wish to investigate.

Stable flame balls have been found to arise only when the Lewis number of a lean reactant is less than unity [5-12]. In some sense, the presence of the non-conducting solid can be thought of as adding to the heat capacity of the system, which in turn can be thought of as reducing an effective Lewis number of the two-phase medium as a whole. The solid might therefore be able to stabilize a gaseous flame ball which would not be stable without the presence of the solid. Apart from making flame balls easier to observe experimentally, a medium of this nature would be safer since it would inhibit the initiation of self-propagating flames. A localized ignition source would tend to create a more benign, stable flame-ball instead.

As a further extension of existing work, we also examine a model for heat loss in the gas that is kept closer to the spirit of Stefan's radiative law. Black-body radiation predicts that the rate of energy loss from any small-volume element increases with the fourth power of absolute temperature. Stefan's law adopts this overall dependence on temperature and also takes the effectiveness of the radiation to be proportional to an emissivity coefficient of the medium, which can change with changing chemical composition. Thus, the burnt gases inside a flame ball might well contain species that are more efficient emitters of radiation than the unburnt gases [15]. The latter circumstance tends to support the use of a model with constant heat loss in the burnt gas and linear heat losses elsewhere.

Even with a uniform emissivity, it can be argued $[6,7]$ that the nonlinear effect of Stefan's law (after removing any component that is linearized about the ambient temperature) is only important where the temperature is high. Since, the burnt gas temperature is almost uniform and temperatures drop fairly quickly into the unburnt region, it follows that a simple and reasonable model for radiative losses would still arise by simply taking them to be constant and present only in the burnt gas, not even being influenced significantly by the small changes in temperature that would be sufficient to bring about order-one changes in the solution when the activation temperature is large. Nevertheless, Stefan's law with uniform emissivity is still highly pertinent, in particular through treating radiation as being nonlinear and distributed continuously over the whole temperature range. We therefore study its effect on the structure and stability of flame balls as well as the effect of its linearized cousin.

The linearized model for radiative losses is simple to analyse which is another significant advantage. In the context of a simple flame-sheet description of the chemistry, with spherical symmetry, exact steady solutions and exact eigenfunction solutions for linear stability can be found. We base our analysis of the problem with linearized heat loss on these exact solutions, not needing therefore to consider a matched asymptotic approach, as in [7], although the interpretation of the dispersion relation for linear stability still requires an asymptotic examination for large activation temperature. However, this is just an examination of algebraic relations rather than the asymptotic solution of differential equations.

An exact approach is not possible for a nonlinear radiative law (Stefan's law). This part of the analysis is therefore more involved, requiring either an iterative or matched asymptotic solution of the relevant differential equations describing the temperature and its eigenfunction. Asymptotic results are obtained, which then provide a useful point for comparison and contrast with the simpler linearized law for heat loss.

The asymptotic work is supplemented by a numerical study of the governing equations, only for the linearized heat-loss model, cast into a finite-difference form. For the numerical work, an Arrhenius law is used to describe the chemistry, since jump conditions at a flame-sheet 
would be more difficult to implement. This provides a framework for comparison of the leading-order asymptotic results (effectively at infinite activation temperature) with solutions at finite activation temperature. The numerical code provides both steady solutions and eigenvalues for spherically symmetric instability. Non-symmetric eigenfunctions $[7,16]$ are not studied in this paper.

\section{Spherically symmetric model}

For a simple one-step reaction, $\mathrm{F} \rightarrow \mathrm{P}$, in which a gaseous reactant $\mathrm{F}$ is converted into a gaseous product $P$ within an unbounded medium that contains a small-volume fraction of porous solid, a suitable dimensional reactive-diffusive model is

$$
\begin{aligned}
& \rho Y_{t}=\rho D\left(Y_{r r}+\frac{2}{r} Y_{r}\right)-W_{\mathrm{F}} \Omega \\
& \rho C_{\mathrm{P}} T_{t}=\lambda\left(T_{r r}+\frac{2}{r} T_{r}\right)+\left(W_{\mathrm{F}} h_{\mathrm{F}}-W_{\mathrm{P}} h_{\mathrm{P}}\right) \Omega+k(\theta-T)+L(T) \\
& q \theta_{t}=k(T-\theta) \\
& \Omega=A \frac{\rho Y}{W_{\mathrm{F}}} \exp \left(-T_{\mathrm{A}} / T\right) H\left(T-T_{\mathrm{c}}\right)
\end{aligned}
$$

in which $t$ is time and $r$ is a radial coordinate about a suitable origin of spherical symmetry. The mass fraction of the reactant is denoted by $Y(t, r)$, the gas temperature by $T(t, r)$ and the solid temperature by $\theta(t, r)$.

The density $\rho$, specific heat $C_{\mathrm{P}}$ and thermal conductivity $\lambda$ of the gas, as well as the diffusion coefficient $D$ of the reactant $\mathrm{F}$, are taken to be constant for simplicity of analysis; the constant-density model is remarkably effective at providing simple but qualitatively accurate predictions of combustion phenomena in which diffusive processes are of predominant importance. The molecular weights of the reactant $\mathrm{F}$ and the product $\mathrm{P}$ are $W_{\mathrm{F}}$ and $W_{\mathrm{P}}$, respectively, with enthalpies of formation $h_{\mathrm{F}}$ and $h_{\mathrm{P}}$. The Arrhenius pre-exponential factor is $A$ and the activation temperature of the reaction is $T_{\mathrm{A}}$. The Heaviside function $H(\cdot)$ ensures that the reaction rate $\Omega$ is actually zero for any temperature below the cut-off temperature $T_{\mathrm{c}}$, guaranteeing chemical equilibrium under ambient conditions for any ambient temperature $T_{\mathrm{a}}$ that is below $T_{\mathrm{c}}$. The model (1) has no unbounded steady solution if the ambient state is reacting, however slowly.

The porous solid is modelled as an inert, homogeneous, porous, non-conducting mesh, or as a relatively non-mobile and uniformly distributed dust. Cases in which the solid phase also conducts heat or, possibly, moves via thermophoresis, are not considered here. Attention is therefore focused on a relatively simple situation in which the primary role of the solid is to exchange heat with the gas, behaving as a kind of heat reservoir.

In this simple continuum model, the parameters $k$ and $q$ govern the exchange of heat between solid and gas. These represent, respectively, the rate of heat transfer (per unit volume of the medium per unit temperature difference) and the heat capacity of the solid (per unit volume of the medium). In broad terms, $k$ should be proportional to the surface area of the solid per unit volume and to the thermal conductivity $\lambda$, with some dependence on the fine-scale geometry or structure of the solid phase - the thermal conductivity of the solid is taken to be high enough and the fine-scale thickness of any part of the solid to be small enough, for heat to be considered to be uniformly distributed across any small element in the solid structure. The volumetric heat capacity $q$ is the mass of solid per unit volume of the medium multiplied by its specific heat capacity. Thus, for a fixed volumetric heat capacity $q$, or mass of solid per 
unit volume, the rate of heat transfer $k$ would increase as the fineness of the mesh containing the solid is increased.

The representation of the system using a continuum of two distinct temperatures, $\theta$ and $T$, assumes that the mesh is fine and interacting thermally with the gas at all points. Of course, both temperatures represent a local average in their respective phases. For the highly nonlinear temperature dependence of the chemistry to be determined by the locally averaged gas temperature $T$, the actual gas temperature at any point should not deviate much from its average value in any suitably small-volume element that is not as small as the fine scale of the mesh. This, in turn, requires that the temperature difference $|T-\theta|$ should be relatively small as, in fact, will be the case for the solutions examined in this paper.

If we also consider the mesh (or dust) to be optically thin, then the remaining term in the model $L(T)$ can be taken to represent radiative heat losses. For the purposes of this paper we consider all such losses to arise from radiation within the gas phase. Stefan's law, for radiative heat exchange with a uniform ambient state, predicts that

$$
L(T)=\varepsilon\left(T_{\mathrm{a}}^{4}-T^{4}\right)
$$

where $\varepsilon$ is the emissivity. We shall study two approximate forms of this law, one of which is linearized differently for burnt and unburnt gases and is therefore more easily analysed. The other, which will be discussed later, retains the nonlinear features of Stefan's law and does not distinguish between the burnt and unburnt media.

Stefan's law (2) linearizes about ambient temperature and about any flame temperature $T_{\mathrm{f}}$ such that

$$
L(T) \approx \begin{cases}-\varepsilon\left(T_{\mathrm{f}}^{4}-T_{\mathrm{a}}^{4}\right)-4 \varepsilon T_{\mathrm{f}}^{3}\left(T-T_{\mathrm{f}}\right) & \text { for } T \approx T_{\mathrm{f}} \\ -4 \varepsilon T_{\mathrm{a}}^{3}\left(T-T_{\mathrm{a}}\right) & \text { for } T \approx T_{\mathrm{a}} .\end{cases}
$$

In the context of a large activation temperature analysis, with the temperature in the burnt gas typically close to $T_{\mathrm{f}}$, the linear term for $T \approx T_{\mathrm{f}}$ will be small compared with the constant term. Thus we can model the linearized losses in the burnt gas as being predominantly constant. For simplicity, in our first model for heat loss, we shall take all losses in the unburnt gas to arise only from the linearization about the ambient state. Thus, a simple linearized model for heat loss becomes

$$
L(T) \approx \begin{cases}-\varepsilon_{\mathrm{b}}\left(T_{\mathrm{f}}^{4}-T_{\mathrm{a}}^{4}\right) & \text { for } \quad T \approx T_{\mathrm{f}} \\ -4 \varepsilon T_{\mathrm{a}}^{3}\left(T-T_{\mathrm{a}}\right) & \text { for } \quad T \approx T_{\mathrm{f}}\end{cases}
$$

where $\varepsilon_{\mathrm{b}}$ is the emissivity of the burnt gas; the different chemical constituents in this region could alter the emissivity.

A flame-ball, satisfying these equations, will also need to satisfy boundary conditions as $r \rightarrow \infty$ of the form

$$
\lim _{r \rightarrow \infty}(Y, T, \theta)=\left(Y_{\mathrm{a}}, T_{\mathrm{a}}, T_{\mathrm{a}}\right)
$$

implicitly requiring that the reaction rate $\Omega$ must be zero for large enough values of $r$ (to avoid the 'cold boundary difficulty' [17] and to ensure that the mixture is chemically stable under ambient conditions). This can be guaranteed by choosing the cut-off temperature $T_{\mathrm{c}}$ to be slightly above $T_{\mathrm{a}}$.

In the absence of the solid phase, these equations are essentially the same as those already studied for flame balls in gases [1-14]. The model (1)-(5) generalizes on this by including the solid phase only as a reservoir of heat that can be exchanged with the gas. The aim of this paper is to study the effect that the presence of this immobile and inert reservoir of heat can 
have on the structure and stability of flame balls. We shall also generalize on previous work by examining heat losses given nonlinearly by Stefan's law (2) as well as by the linearized approximation (4).

\subsection{Dimensionless model}

A dimensionless form of the model arises if we redefine all variables, such that

$$
\begin{array}{cc}
T=T_{\mathrm{a}}+\left(T_{\mathrm{f}}-T_{\mathrm{a}}\right) T^{\prime} & \theta=T_{\mathrm{a}}+\left(T_{\mathrm{f}}-T_{\mathrm{a}}\right) \theta^{\prime} \quad Y=Y_{\mathrm{a}} Y^{\prime} \\
t=t_{\mathrm{s}} t^{\prime} \quad r=l_{\mathrm{s}} r^{\prime} & L\left(T_{\mathrm{a}}+\left(T_{\mathrm{f}}-T_{\mathrm{a}}\right) T^{\prime}\right)=\frac{T_{\mathrm{f}}-T_{\mathrm{a}}}{l_{\mathrm{s}}^{2} / \lambda} L^{\prime}\left(T^{\prime}\right)
\end{array}
$$

(subsequently dropping the primes). If we select the rescaling so as to define

$$
\begin{aligned}
& T_{\mathrm{f}}=T_{\mathrm{a}}+\frac{\left(W_{\mathrm{F}} h_{\mathrm{F}}-W_{\mathrm{P}} h_{\mathrm{P}}\right)}{W_{\mathrm{F}} C_{\mathrm{P}}} \frac{Y_{\mathrm{a}}}{L e} \quad t_{\mathrm{s}}=\frac{\rho C_{\mathrm{P}}}{\lambda} l_{\mathrm{s}}^{2} \quad l_{\mathrm{s}}^{2}=\frac{1}{2} \beta^{2} \frac{D}{A} \mathrm{e}^{T_{\mathrm{A}} / T_{\mathrm{f}}} \\
& L e=\frac{\lambda}{\rho C_{\mathrm{P}} D} \quad \mu=\frac{q}{\rho C_{\mathrm{P}}} \quad \beta=\frac{T_{\mathrm{A}}\left(T_{\mathrm{f}}-T_{\mathrm{a}}\right)}{T_{\mathrm{f}}^{2}} \quad \gamma=\frac{T_{\mathrm{f}}-T_{\mathrm{a}}}{T_{\mathrm{f}}} \quad \tau=\frac{q}{k t_{\mathrm{s}}} \\
& b=\beta \frac{\varepsilon_{\mathrm{b}} l_{\mathrm{s}}^{2}}{\lambda} \frac{T_{\mathrm{f}}^{4}-T_{\mathrm{a}}^{4}}{T_{\mathrm{f}}-T_{\mathrm{a}}} \quad a^{2}=\beta^{2} \frac{4 \varepsilon T_{\mathrm{a}}^{3} l_{\mathrm{s}}^{2}}{\lambda}
\end{aligned}
$$

then the governing equations, with the linearized model (4) for heat loss, take on the dimensionless form

$$
\begin{aligned}
& \text { LeY } Y_{t}=Y_{r r}+\frac{2}{r} Y_{r}-\Omega \\
& T_{t}+\mu \theta_{t}=T_{r r}+\frac{2}{r} T_{r}+\Omega+L \\
& \tau \theta_{t}=T-\theta \\
& \Omega=\frac{1}{2} \beta^{2} Y \exp \left(\frac{\beta(T-1)}{1+\gamma(T-1)}\right) H\left(T-T_{\mathrm{c}}\right) \\
& \lim _{r \rightarrow \infty}(Y, T, \theta)=(1,0,0) \quad L=- \begin{cases}b / \beta & \text { for } T \approx 1 \\
a^{2} T / \beta^{2} & \text { for } T \not z 1 .\end{cases}
\end{aligned}
$$

The parameter $L e$ is the Lewis number and $\beta$ is the Zeldovich number. For a realistic model of combustion $\beta$ must be large, a useful key for asymptotic solution of many combustion problems.

The parameter $\mu$ is the dimensionless heat capacity of the solid phase and $\tau$ is a dimensionless time-scale for the transfer of heat between the gas and solid phases. The parameters $a$ and $b$ (both taken to be positive, or possibly zero) represent linearized effects of heat loss in the unburnt and burnt gases through radiation. It can be noted that the factors of $\beta^{-1}$ and $\beta^{-2}$ in the expression for $L$ ensure that these losses are relatively weak in both parts of the combustion process for order-one values of $a$ and $b$. Taking the radiative losses per unit volume to be weaker by a factor of the order of $\beta^{-1} T$ in the unburnt gas is consistent with the nonlinearly disproportionate reduction that occurs through the gases being colder there.

We shall proceed by assuming that $\mu, \tau, a$ and $b$ are all of order one, while $\beta$ is large. Taking $\mu$ to be of order-one implicitly assumes that the mass of solid per unit volume is similar to that of the gas. There would therefore be relatively little solid present per unit volume, requiring a volume fraction of the order of $10^{-3}$ under normal atmospheric conditions. The corresponding solid matrix would therefore be relatively light, rather like a fine suspension of dust particles that are also assumed, in this paper, to be relatively immobile. 
However, although the analysis proceeds by taking $\mu$ to be of order one, the results are still valid when $\mu$ is large. Thus the mass of the solid does not have to be particularly small per unit volume of the medium. In practice, a rigid porous mesh would tend to be much heavier than the gas within it, making the value of $\mu$ correspondingly large.

\subsection{Exchange of heat with the solid phase}

It is worth noting immediately that if $T(t, r)$ is a solution for $T$, then the solid temperature $\theta$ is determined by

or

$$
\theta(t, r)=\mathrm{e}^{\left(t_{0}-t\right) / \tau} \theta_{0}(r)+\int_{t_{0}}^{t} \frac{\mathrm{e}^{(\zeta-t) / \tau}}{\tau} T(\zeta, r) \mathrm{d} \zeta
$$

$$
\theta(t, r)=\int_{-\infty}^{t} \frac{\mathrm{e}^{(\zeta-t) / \tau}}{\tau} T(\zeta, r) \mathrm{d} \zeta
$$

where $\theta$ has the initial value $\theta=\theta_{0}(r)$ at the time $t=t_{0}$. After a while, when $t-t_{0} \gg \tau$, the effect of the initial condition is effectively forgotten and the second result for $\theta$ is a more appropriate solution. Since $\int_{-\infty}^{t} \frac{1}{\tau} \mathrm{e}^{(\zeta-t) / \tau} \mathrm{d} \zeta=1$, the latter solution shows that the value adopted by $\theta$ at any point is a weighted average of the earlier values of $T$ at that point.

In the limiting case when $\tau \rightarrow \infty$ the solid temperature stays constant and does not interact with the gas. The equations for the scaled mass fraction and gas temperature are then the same as they would be with no solid present at all, namely

$$
L e Y_{t}=Y_{r r}+\frac{2}{r} Y_{r}-\Omega \quad T_{t}=T_{r r}+\frac{2}{r} T_{r}+\Omega+L .
$$

Of course, the same equations hold in the limiting case when the solid has no heat capacity, $\mu=0$, as they would do if it was simply not present. At the opposite extreme, in the limiting case when $\tau \rightarrow 0$ the gas and solid temperatures are always equal, $\theta \equiv T$. Rescaling time so that $t=(1+\mu)$ t, the equations for the scaled mass fraction $Y$ and temperature $T$ now become

$$
\frac{L e}{1+\mu} Y_{\mathrm{t}}=Y_{r r}+\frac{2}{r} Y_{r}-\Omega \quad T_{\mathrm{t}}=T_{r r}+\frac{2}{r} T_{r}+\Omega+L
$$

which shows that the model is then equivalent to the model with no solid present, but with the Lewis number replaced by $L e /(1+\mu)$. This is because, when $\tau=0$, the specific heat capacity $C_{\mathrm{P}}$ is effectively increased by the factor $1+\mu$. The heat capacity of the solid augments that of the gas and, when $\mu$ is very large, the 'effective' Lewis number $L e /(1+\mu)$ would tend to be very small. To some extent therefore, even when $\tau \neq 0$, the presence of the solid should have an effect similar to that of reducing the Lewis number of the gas, at least in some cases.

\subsection{Flame-sheet model}

Assuming that there is a reaction sheet around a location $r=R(t)$, we can focus attention on a suitable inner region by rescaling such that

$$
r=R+\beta^{-1} \xi \quad Y=\beta^{-1} \psi \quad T=1-\beta^{-1} \phi .
$$

The leading-order equations for $\psi(t, \xi)$ and $\phi(t, \xi)$, as $\beta \rightarrow \infty$, become

$$
\psi_{\xi \xi}=\phi_{\xi \xi}=\frac{1}{2} \psi \mathrm{e}^{-\phi} .
$$


In the burnt gases where $r<R$, the value of $\psi$ should approach zero as $\beta \rightarrow \infty$, while gradients in temperature will be relatively small (also tending to zero as $\beta \rightarrow \infty$ ). Suitable boundary conditions are therefore

$$
\lim _{\xi \rightarrow-\infty}\left(\psi, \phi_{\xi}\right)=(0,0)
$$

from which the partial solution

$$
\phi=\widehat{\phi}+\psi \quad \psi_{\xi}^{2}=\mathrm{e}^{-\widehat{\phi}}\left(1-(1+\psi) \mathrm{e}^{-\psi}\right)
$$

can be obtained, with $\widehat{\phi}=\widehat{\phi}(t)$. A further integration would determine the spatial structure of $\psi(t, \xi)$ and $\phi(t, \xi)$, modulo arbitrary translations in $\xi$, parametrized by the time-dependent function $\widehat{\phi}(t)$.

In terms of the outer variables, $Y$ and $T$, this solution has the form

$$
Y+T=\widehat{T}+\mathrm{O}\left(\beta^{-2}\right) \quad Y_{r}^{2}=\mathrm{e}^{\beta(\widehat{T}-1)}\left(1-(1+\beta Y) \mathrm{e}^{-\beta Y}\right)+\mathrm{O}\left(\beta^{-1}\right)
$$

which can only be asymptotically valid if $r=R+\beta^{-1} \xi$ tends to $R$ as $\beta \rightarrow \infty$, thus providing matching conditions for the solution of the outer problem if it is also assumed that $1 \ll|\xi| \ll \beta$. By suitably selecting the value of $R$, this leads to the following jump conditions in the values and gradients of $Y$ and $T$ across the location $r=R^{ \pm}$:

$$
[T]=[Y]=Y=0 \quad\left[Y_{r}\right]=-\left[T_{r}\right]=\mathrm{e}^{\beta(T-1) / 2}
$$

evaluated at the flame sheet $r=R$. Higher-order effects have been neglected in writing out these jump conditions. Away from the flame sheet, the reaction rate term is negligible so that for $r \neq R$ the equations to be satisfied are

$$
\begin{aligned}
& \operatorname{LeY}_{t}=Y_{r r}+\frac{2}{r} Y_{r} \\
& T_{t}+\mu \theta_{t}=T_{r r}+\frac{2}{r} T_{r}- \begin{cases}b / \beta & \text { if } r<R \\
a^{2} T / \beta^{2} & \text { if } r>R\end{cases} \\
& \tau \theta_{t}=T-\theta \\
& \lim _{r \rightarrow \infty}(Y, T, \theta)=(1,0,0)
\end{aligned}
$$

at least in the case of the linearized model for heat loss. In this form of the model, the different heat-loss terms are now explicitly distinguished between the burnt region $(r<R)$ and the unburnt region $(r>R)$.

In principle, the jump conditions (17) can be extended to higher order, involving other effects such as the nonlinear form of the exponent in (8), curvature, propagation speed $\frac{\mathrm{d} R}{\mathrm{~d} t}$ and temperature gradient in the burnt gas [18]. However, these effects would normally make little overall difference and so we shall proceed using the jump conditions as given in equations (17) to represent the entire effect of the chemistry, taking them to be correct to all orders as $\beta \rightarrow \infty$.

\subsection{Stefan's law for heat loss}

A dimensionless model for heat loss that keeps more closely to the spirit of Stefan's radiative law, applied uniformly throughout the medium, arises if the dimensional heat loss expression (2) is written in dimensionless form as

$$
L(T)=-\frac{b}{\beta}\left(T^{4}+\frac{4 T_{\mathrm{a}}}{\gamma T_{\mathrm{f}}} T^{3}+\frac{6 T_{\mathrm{a}}^{2}}{\gamma^{2} T_{\mathrm{f}}^{2}} T^{2}\right)-\frac{a^{2}}{\beta^{2}} T \quad \text { with } \quad b=\beta \frac{\varepsilon l_{\mathrm{s}}^{2}}{\lambda}\left(T_{\mathrm{f}}-T_{\mathrm{a}}\right)^{3} .
$$


Note that the font used here for the symbol $b$ is distinct from that of the parameter $b$ used in the models (8) and (18). It can also be noted that this is the exact dimensionless form of (2), with no further approximation, and that the definition of $b$ is very similar to that of $b$ in (7). The two should therefore have similar magnitudes, given approximately by $\beta \varepsilon l_{\mathrm{s}}^{2} T_{\mathrm{f}}^{3} / \lambda$ whenever $T_{\mathrm{f}}^{4} \gg T_{\mathrm{a}}^{4}$. When this is true, the nonlinear terms in the expression (19) for $L(T)$ are also dominated by the fourth power of $T$. If the other nonlinear terms are neglected, then an alternative and relatively simple model equation for the gas temperature becomes

$$
T_{t}+\mu \theta_{t}=T_{r r}+\frac{2}{r} T_{r}-b T^{4} / \beta-a^{2} T / \beta^{2}
$$

which would be valid for any value of $r \neq R$, the same equation applying in both burnt and unburnt gases. In cases where the temperature change through the flame ball is not large, a linear heat loss law would be suitable everywhere. This would correspond to having $b=0$.

\subsection{Numerical solutions}

As well as the asymptotic studies that are outlined in section 3 , equations (8) were solved numerically. In order to do this on a finite grid, the transformation $r=\beta \tan (x / \beta)$ was used so that only a finite domain $0 \leqslant x \leqslant \frac{\pi}{2} \beta$ needed to be discretized. This was done using a standard finite-difference approach, with continuation, for which Newton iteration was found to produce converged results with 8000 evenly spaced grid points, even when taking $\beta=100$. Eigenvalues for the linear stability of steady solutions were calculated using a shifted inverse power method. An additional modification, to allow the cold-boundary cut-off to be described more smoothly than it would be using a Heaviside function, was made by defining

$$
\Omega=\max \left\{\frac{1}{2} \beta^{2} Y\left(\mathrm{e}^{\beta(T-1) / 3}-\mathrm{e}^{1-\beta(2 T+1) / 3}\right)^{3}, 0\right\} .
$$

This is practically the same as the definition in (8), having exactly the same asymptotic behaviour for large $\beta$ with $T>\mathrm{O}\left(\beta^{-1}\right)$, but it is continuously differentiable so that it is more amenable to numerical solution using Newton iteration. The linearized heat loss model in (8) was rewritten as

$$
L(T)=-\frac{b / \beta+a^{2} T \beta^{2} r^{4} Y^{4}}{1+\beta^{4} r^{4} Y^{4}}
$$

which acts as an effective switch between the heat-loss definitions in the burnt and unburnt gases, while also being continuously differentiable. Numerical solutions were only examined for the linearized heat loss model of equations (8) and not for the continuous, nonlinear heat loss law in (20). Finally, the radius $R$ was estimated in any numerical solution using one or both of the alternative formulae

$$
R_{\mathrm{I}}=\int_{0}^{\infty} \frac{\mathrm{d} r}{1+\beta^{4} r^{4} Y^{4}} \quad \text { and } \quad R_{\mathrm{F}}=r(1-Y) \text { for } T \leqslant \beta^{-1}
$$

the latter being more suitable in completely steady solutions for any value of $Y$ that is not too close to zero or unity, say a value of around $\frac{1}{2}$.

\section{Flame balls and their stability}

We can now examine the effect on spherical flame balls of the presence of an inert solid that contributes only through an exchange of heat with the reacting gas. 


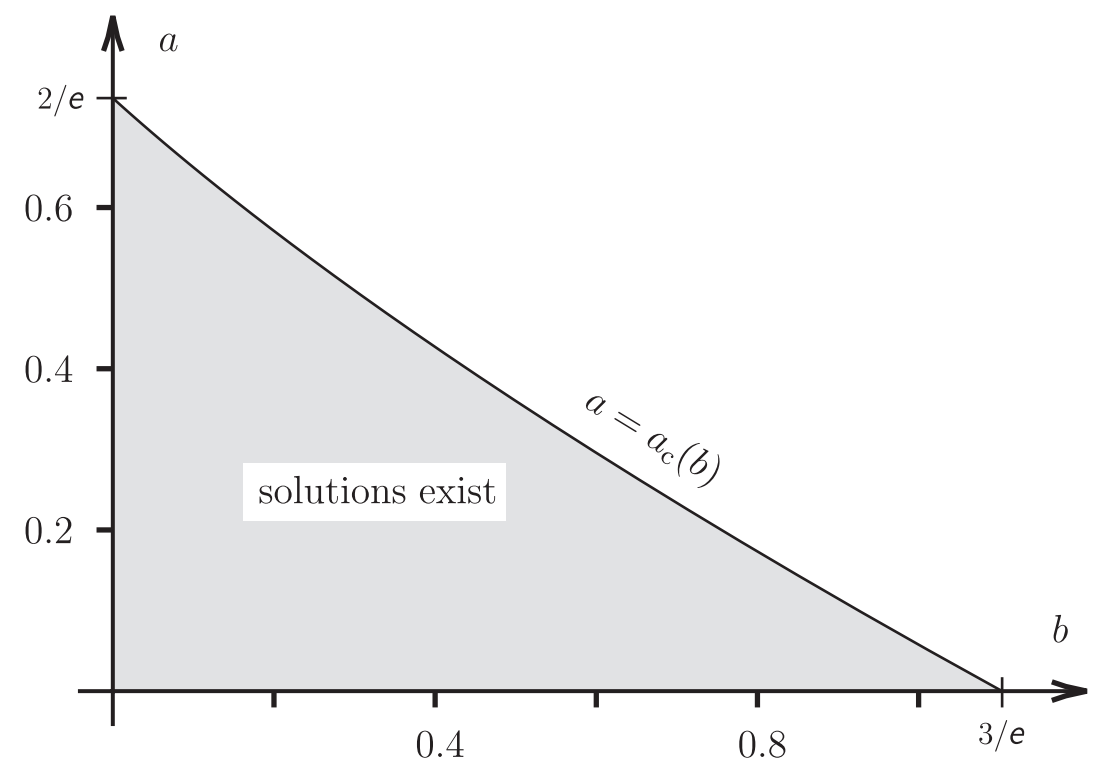

Figure 1. The range of values of the heat-loss parameters $a$ and $b$ within which solutions exist, namely $0 \leqslant a \leqslant a_{\mathrm{c}}(b)$, with $b \geqslant 0$.

\subsection{Linearized heat loss.}

We shall firstly examine the model (17) and (18) in which the heat loss term $L(T)$ is linearized and assigned the constant value $b / \beta$ in the burnt gas. The steady solution is a natural starting point for this.

3.1.1. Steady solutions. If there is no time dependence, then we can immediately note that the solid and gas temperatures are the same, $\theta \equiv T$. The model (18) is then precisely the same as it would be if there were no solid phase present at all, namely, for $r \neq R$, with $\partial_{t} \equiv 0$

$$
Y_{r r}+\frac{2}{r} Y_{r}=0 \quad T_{r r}+\frac{2}{r} T_{r}= \begin{cases}b / \beta & \text { if } \quad r<R \\ a^{2} T / \beta^{2} & \text { if } \quad r>R\end{cases}
$$

along with the jump conditions at $r=R$ and the conditions at infinity

$$
[T]=[Y]=Y=0 \quad\left[Y_{r}\right]=-\left[T_{r}\right]=\mathrm{e}^{\beta(T-1) / 2} \quad \lim _{r \rightarrow \infty}(Y, T, \theta)=(1,0,0) .
$$

Steady solutions in which $R(t) \equiv S$ have the form

$$
\begin{aligned}
& Y=Y_{0}(r)= \begin{cases}0 & \text { if } r<S \\
1-S / r & \text { if } r>S\end{cases} \\
& \theta=T=T_{0}(r)= \begin{cases}\widehat{T}+\frac{b}{\beta} \frac{r^{2}-S^{2}}{6} & \text { if } \quad r<S \\
\widehat{T} \frac{S}{r} \mathrm{e}^{a(S-r) / \beta} & \text { if } \quad r>S\end{cases}
\end{aligned}
$$



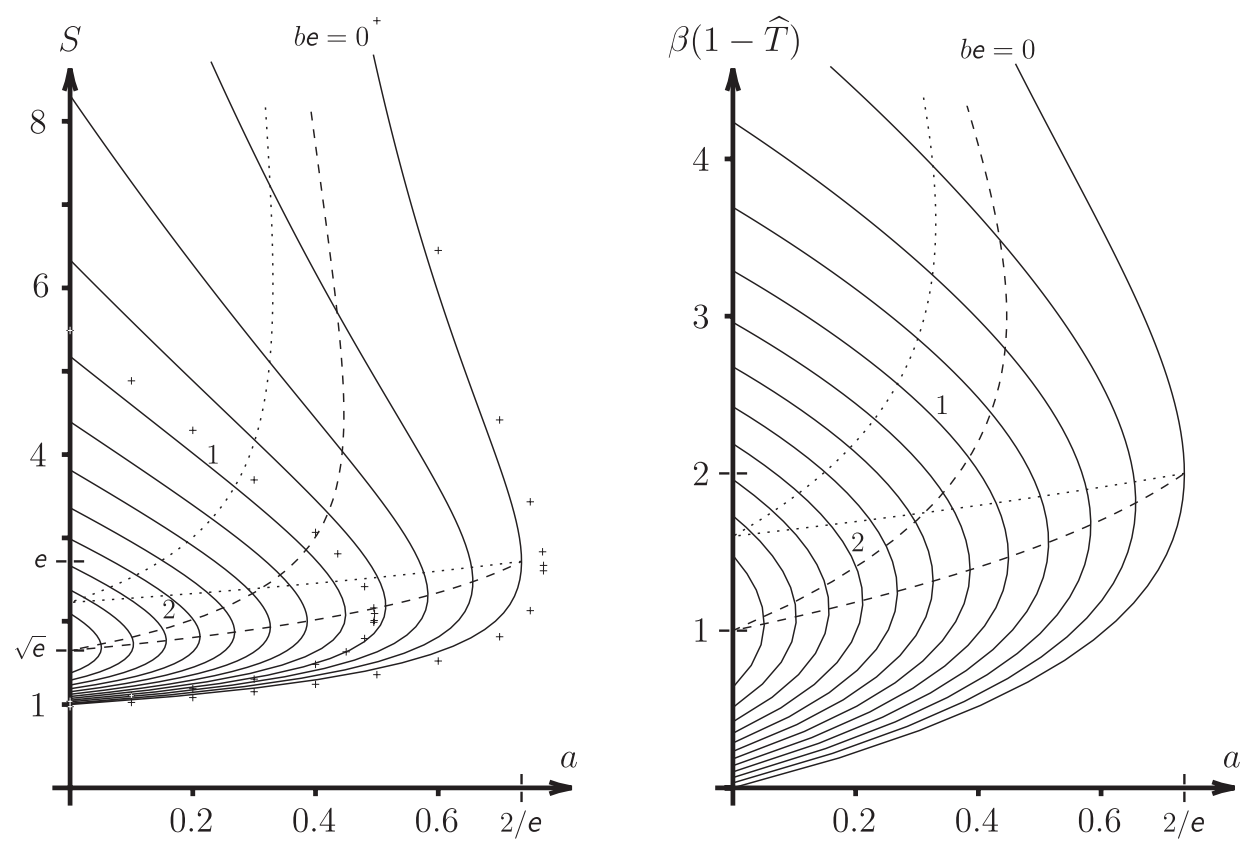

Figure 2. Variations with $a$ of steady radius $S$ (left) and temperature (right), shown as $\beta(1-\widehat{T})$, at fixed values of $b$ between 0 and $3 / \mathrm{e}$. The small + symbols are numerical results calculated for $b=0$ and $b \mathrm{e}=1$ with $\beta=100$. The lower broken curve follows the critical path $a=a_{\mathrm{c}}(b)$, corresponding to $G=1$ in figure 5 and the upper broken curve corresponds to $G=0$. The lower dotted curve corresponds to $G=1$ in figure 7 and the upper dotted curve corresponds to $G=0$.

satisfying the continuity conditions, $[T]=[Y]=Y=0$, at $r=S$ and the conditions at infinity. The condition that $\left[Y_{r}\right]=-\left[T_{r}\right]$ leads to

$$
\widehat{T}=\frac{1-\frac{1}{3} b S^{2} / \beta}{1+a S / \beta}=1-\beta^{-1}\left(a S+\frac{1}{3} b S^{2}\right)+\mathrm{O}\left(\beta^{-2}\right) .
$$

Since $T=\widehat{T}$ at $r=S$, the condition $\left[Y_{r}\right]=\mathrm{e}^{\beta(\widehat{T}-1) / 2}$ then leads to

$$
S=\exp \left(\frac{1}{2} a S+\frac{1}{6} b S^{2}\right)+\mathrm{O}\left(\beta^{-1}\right)
$$

or

$$
\beta(1-\widehat{T})=\ln S^{2}=a S+\frac{1}{3} b S^{2}+\mathrm{O}\left(\beta^{-1}\right)
$$

Solutions exist, as $\beta \rightarrow \infty$, for $a \leqslant a_{\mathrm{c}}(b)$, or equivalently $b \leqslant b_{\mathrm{c}}(a)$; a path of fold bifurcations, at which $a S+\frac{1}{3} b S^{2}$ is tangent to $\ln S^{2}$, terminates the solutions. The critical boundary $a=a_{\mathrm{c}}(b)$, or $b=b_{\mathrm{c}}(a)$, is provided by the curve

$a=2 \frac{\ln R_{\mathrm{c}}^{2}-1}{R_{\mathrm{c}}} \in[0,2 / \mathrm{e}] \quad b=3 \frac{2-\ln R_{\mathrm{c}}^{2}}{R_{\mathrm{c}}^{2}} \in[0,3 / \mathrm{e}] \quad$ for $\quad R_{\mathrm{c}} \in\left[\mathrm{e}^{1 / 2}, \mathrm{e}\right]$

parametrized by the critical radius of a steady flame ball $S=R_{\mathrm{c}}$ that is found on the critical boundary. The range of values of $(a, b)$ within which solutions exist is shown in figure 1 . Within this range there are two solutions arising at two different radii. One of these solutions moves away to infinity as both $a$ and $b$ tend to zero, leaving only a solution with $S=1$ and 

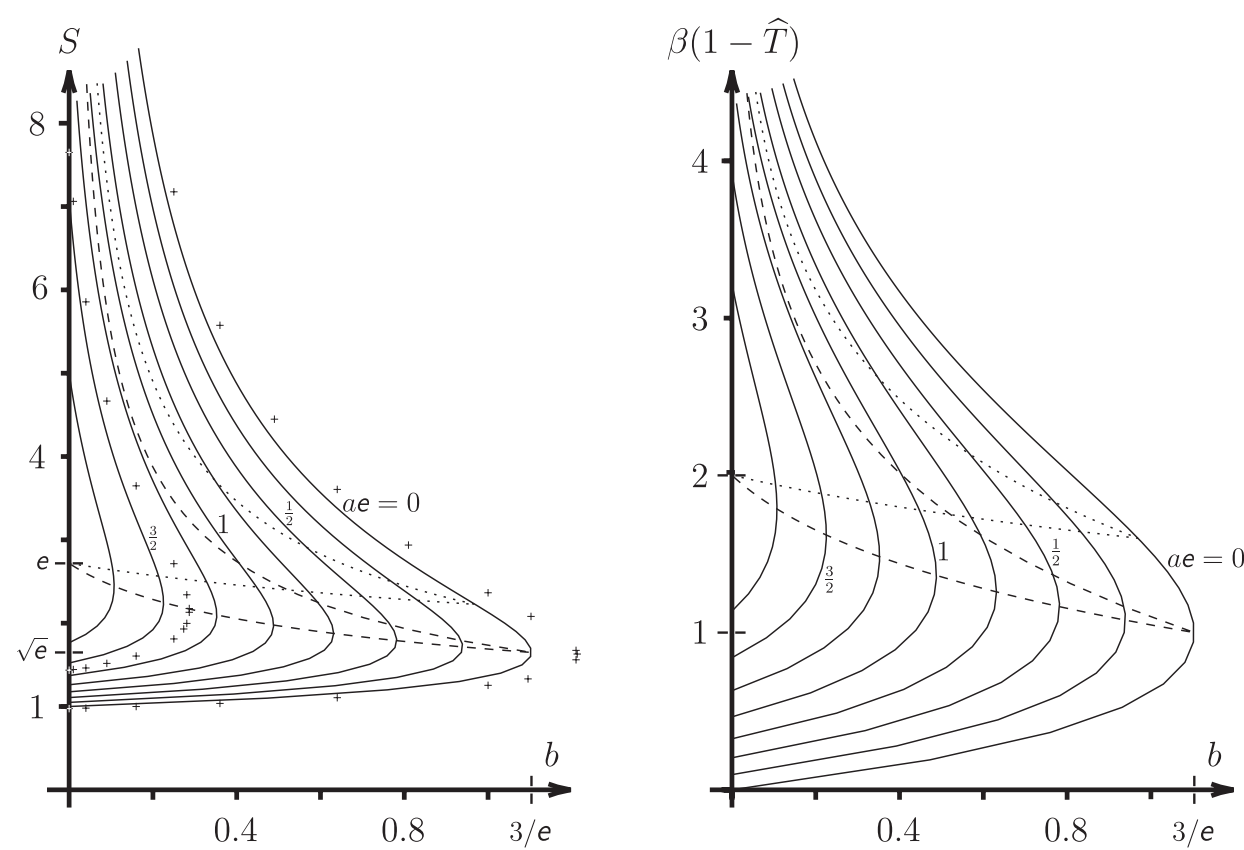

Figure 3. Variations with $b$ of steady radius $S$ (left) and temperature (right), shown as $\beta(1-\widehat{T})$, at fixed values of $a$ between 0 and $2 / \mathrm{e}$. The small + symbols are numerical results calculated for $a=0$ and $a \mathrm{e}=\frac{3}{2}$ with $\beta=100$. The lower broken curve follows the critical path $a=a_{\mathrm{c}}(b)$, corresponding to $G=1$ in figure 5 and the upper broken curve corresponds to $G=0$. The lower dotted curve corresponds to $G=1$ in figure 7 and the upper dotted curve corresponds to $G=0$.

$\widehat{T}=1$. These are the dimensionless radius and temperature of a Zeldovich flame ball [1] without any heat losses.

Figures 2 and 3 illustrate the dependence of radius and temperature on $a$ at fixed values of $b$, and on $b$ at fixed values of $a$, respectively. These figures also present some numerically calculated steady flame radii at $a=0, a \mathrm{e}=\frac{3}{2}, b=0$ and $b \mathrm{e}=1$, marked by the small + symbols. The values plotted were all calculated for a Zeldovich number of $\beta=100$. Normally, a Zeldovich number of $\beta=10$ or 20 is considered reasonably large, and yet, even for such a large value of $\beta$, the numerical results deviate from the corresponding asymptotic curves by about $10 \%$.

Table 1 gives an idea of the dependence of solutions on $\beta$, by looking at the two main fold bifurcation points for $b=0$ and for $a=0$. At $\beta=50$ and 100 these are still relatively far from the asymptotic value when $\beta=\infty$, although certainly approaching it as $\beta$ increases. This demonstrates that the asymptotic solutions may not be all that good at describing solutions in which $\beta$ is only moderately large. In our calculations, the values of $a$ and $b$ at the fold bifurcations were found to arise at a distance of the order of one away from their asymptotic values when $\beta \approx 30$. The fold bifurcation at $a=0$ was found to disappear altogether (implying no multivaluedness of solutions) when $\beta$ was between 20 and 30 in value, and the same probably happens for the bifurcation at $b=0$, although this was not investigated.

This paper is mainly concerned with investigating flame balls in the asymptotic limit $\beta \rightarrow \infty$ and so the remaining numerical results that are to be presented were all calculated by taking $\beta=100$. 
Table 1. Numerically calculated values of $a$ (with $b=0$ ) and $b$ (with $a=0$ ) at the fold bifurcation points for $\beta=50$ and 100 . The values shown for $\beta=\infty$ are the corresponding asymptotic result.

\begin{tabular}{rll}
\hline$\beta$ & $a($ at $b=0)$ & $b($ at $a=0)$ \\
\hline 50 & 0.8102 & 1.3532 \\
100 & 0.7716 & 1.2129 \\
$\infty$ & 0.7358 & 1.1036 \\
\hline
\end{tabular}

3.1.2. Spherically symmetric stability. We can now examine the linear stability of the steady solutions (26), to spherically symmetric disturbances, by writing

$$
\begin{array}{ll}
R=S+\mathrm{e}^{\lambda t} & T=T_{0}+\mathrm{e}^{\lambda t} \Gamma(r) \\
Y=Y_{0}+\mathrm{e}^{\lambda t} y(r) & \theta=T_{0}+\mathrm{e}^{\lambda t} \Theta(r)
\end{array}
$$

taking $\mathrm{e}^{\lambda t}$ to be infinitesimally small, for which the equations to be satisfied are

$$
\begin{aligned}
& y^{\prime \prime}+\frac{2}{r} y^{\prime}=\lambda \text { Ley } \\
& \Gamma^{\prime \prime}+\frac{2}{r} \Gamma^{\prime}=\lambda\left(1+\frac{\mu}{1+\lambda \tau}\right) \Gamma+ \begin{cases}0 & \text { if } \quad r<S \\
a^{2} \Gamma / \beta^{2} & \text { if } \quad r>S\end{cases} \\
& (1+\lambda \tau) \Theta=\Gamma \\
& \lim _{r \rightarrow \infty}(y, \Gamma, \Theta)=(0,0,0)
\end{aligned}
$$

with $y \equiv 0$ for $r<S$. The jump conditions at $r=S^{ \pm}$

$$
\left[T_{0}^{\prime}+\Gamma\right]=\left[Y_{0}^{\prime}+y\right]=0 \quad\left[Y_{0}^{\prime \prime}+y^{\prime}\right]=-\left[T_{0}^{\prime \prime}+\Gamma^{\prime}\right]=\frac{\beta}{2 S}\left(T_{0}^{\prime}+\Gamma\right)
$$

are arrived at by Taylor expansion of the conditions (17) about $R=S$. It can be noted from the solutions for a steady flame ball and/or equations (18) that we also have

$\left[Y_{0}^{\prime}\right]=-\left[T_{0}^{\prime}\right]=1 / S \quad\left[Y_{0}^{\prime \prime}\right]=-2 / S^{2} \quad\left[T_{0}^{\prime \prime}\right]=2 / S^{2}-b / \beta+a^{2} \widehat{T} / \beta^{2}$

so that

$[\Gamma]=-y\left(S^{+}\right)=1 / S \quad b / \beta-\left[\Gamma^{\prime}\right]+\mathrm{O}\left(\beta^{-2}\right)=y^{\prime}\left(S^{+}\right)=\frac{2}{S^{2}}+\frac{\beta}{2 S}\left(T_{0}^{\prime}+\Gamma\right)$

with errors that are at most of order $\beta^{-2}$.

Defining

$$
\delta^{2}=\lambda+\frac{\mu \lambda}{1+\lambda \tau} \quad \kappa^{2}=\lambda+\frac{\mu \lambda}{1+\lambda \tau}+\frac{a^{2}}{\beta^{2}} \quad v^{2}=\lambda L e
$$

the equations for $y, \Gamma$ and $\Theta$ can be solved to give

$$
\begin{aligned}
& y=\left\{\begin{array}{lr}
0 & \text { if } r<S \\
\widehat{y} \frac{S}{r} \mathrm{e}^{v(S-r)} & \text { if } r>S
\end{array}\right. \\
& \Gamma=(1+\lambda \tau) \Theta= \begin{cases}\widehat{\Gamma}_{\mathrm{b}} \frac{S}{r} \frac{\sinh (\delta r)}{\sinh (\delta S)} & \text { if } r<S \\
\widehat{\Gamma}_{\mathrm{a}} \frac{S}{r} \mathrm{e}^{\kappa(S-r)} & \text { if } r>S\end{cases}
\end{aligned}
$$


provided we assume, without loss of generality, that $\operatorname{Re}(v) \geqslant 0$ and $\operatorname{Re}(\kappa) \geqslant 0$. The various jump conditions now reveal that

$\widehat{\Gamma}_{\mathrm{a}}-\widehat{\Gamma}_{\mathrm{b}}=-\widehat{y}=1 / S$

$\widehat{\Gamma}_{\mathrm{a}}\left(S+\kappa S^{2}\right)+\widehat{\Gamma}_{\mathrm{b}}\left(\delta S^{2} \operatorname{coth}(\delta S)-S\right)+\frac{b}{\beta} S^{2}=-\widehat{y}\left(S+v S^{2}\right)=2+\frac{1}{6} b S^{2}+\frac{1}{2} \beta S \widehat{\Gamma}_{\mathrm{b}}$

after ignoring terms that are of the order of $\beta^{-2}$ or less. From this it can be seen that

$\widehat{y}=-\frac{1}{S} \quad \widehat{\Gamma}_{\mathrm{a}}=\widehat{\Gamma}_{\mathrm{b}}+\frac{1}{S}=\frac{\delta \operatorname{coth}(\delta S)+v-\frac{b}{\beta} S}{\delta S \operatorname{coth}(\delta S)+\kappa S}=\frac{\beta-2-\frac{1}{3} b S^{2}+2 v S}{\beta S}$

revealing the dispersion relation

$$
\beta(\kappa-v)=-b S+\left(2+\frac{1}{3} b S^{2}-2 v S\right)(\delta \operatorname{coth}(\delta S)+\kappa)
$$

or, substituting for $\delta, \kappa$ and $v$

$$
\begin{aligned}
\left(\lambda+\frac{\mu \lambda}{1+\lambda \tau}+\right. & \left.\frac{a^{2}}{\beta^{2}}\right)^{1 / 2}-(\lambda L e)^{1 / 2}=-b S / \beta+\beta^{-1}\left(2+\frac{1}{3} b S^{2}-2(\lambda L e)^{1 / 2} S\right) \\
& \times\left(\left(\lambda+\frac{\mu \lambda}{1+\lambda \tau}\right)^{1 / 2} \operatorname{coth}\left(\left(\lambda+\frac{\mu \lambda}{1+\lambda \tau}\right)^{1 / 2} S\right)+\left(\lambda+\frac{\mu \lambda}{1+\lambda \tau}+\frac{a^{2}}{\beta^{2}}\right)^{1 / 2}\right)
\end{aligned}
$$

in which the square roots on the left must be assumed to have a non-negative real part for the eigenfunctions to be bounded at infinity. It can be noted that no assumption that would restrict the size of $\mu$ has been invoked in arriving at this result. We now need to solve the dispersion relation (40) to determine all possible eigenvalues $\lambda$.

Large eigenvalues. Considering firstly values of $\lambda$ that may be large as $\beta \rightarrow \infty$ produces the leading-order form of the dispersion relation, for any value of $\mu \geqslant 0$,

$$
\left(\lambda+\frac{\mu \lambda}{1+\lambda \tau}\right)^{1 / 2}=\beta \frac{L e^{1 / 2}-[1+\mu /(1+\lambda \tau)]^{1 / 2}}{4 L e^{1 / 2} S}
$$

in which the heat loss parameters $a$ and $b$ play no part. In this form, the dispersion relation allows for the possibility that the dimensionless heat-transfer time $\tau$ could be small, having $\lambda \tau=\mathrm{O}(1)$. Since $\lambda^{1 / 2}$ has positive real part in this formula, it follows that large values of $\lambda$ appear only if $L e>1+\mu /(1+\lambda \tau)$. There are three distinct ranges of solution, depending on the magnitude of $\tau$ :

$$
\lambda= \begin{cases}\beta^{2} \frac{\left(L e^{1 / 2}-(1+\mu)^{1 / 2}\right)^{2}}{16 L e S^{2}(1+\mu)} & \text { for } \quad L e>1+\mu \quad \tau=\mathrm{o}\left(\beta^{-2}\right) \\ \beta^{2} \frac{\left(L e^{1 / 2}-[1+\mu /(1+\lambda \tau)]^{1 / 2}\right)^{2}}{16 L e S^{2}[1+\mu /(1+\lambda \tau)]} & \text { for } \quad L e>1+\frac{\mu}{1+\lambda \tau} \quad \tau=\mathrm{O}\left(\beta^{-2}\right) \\ \beta^{2} \frac{\left(L e^{1 / 2}-1\right)^{2}}{16 L e S^{2}} & \text { for } \quad L e>1 \quad \tau \gg \beta^{-2} \mu .\end{cases}
$$

In general, these formulae show that if $\tau$ is of the order of $\beta^{-2}$, then positive and order $\beta^{2}$ values of $\lambda$ arise for large enough values of the Lewis number, namely for $L e>L e_{\mathrm{c}}\left(\mu, \beta^{2} \tau, S\right)$. The threshold Lewis number $L e_{\mathrm{c}}$ decreases from $1+\mu$, as $\beta^{2} \tau \rightarrow 0$, to unity, as $\beta^{2} \tau \rightarrow \infty$, and increases to infinity if $\mu$ increases such that $\mu \beta^{-2} / \tau \rightarrow \infty$. 
Thus it can be seen that for values of $\tau$ that are small compared with $\beta^{-2}$, the system obtains a large positive eigenvalue only if $L e>1+\mu$. If $\tau$ increases, the threshold value of the Lewis number, above which a strong instability appears, decreases to unity. Large positive eigenvalues do not arise at lower Lewis numbers. Moreover, if $\mu$ is increased to large enough values, and in particular if it increases such that $\mu / \tau \gg \beta^{2}$, then no large unstable eigenvalue is found at any order-one Lewis number.

Order-one eigenvalues. Assuming that $\lambda$ is of the order of one, provides the leading-order form of the dispersion relation, also for any value of $\mu \geqslant 0$

$$
\left(\lambda+\frac{\mu \lambda}{1+\lambda \tau}\right)^{1 / 2}-(\lambda L e)^{1 / 2}=0
$$

in which, once again, the heat loss parameters $a$ and $b$ do not appear. Provided $\tau \neq 0$ and $\tau \neq \infty$, this equation has only one non-zero root

$$
\lambda=\frac{1+\mu-L e}{\tau(L e-1)}
$$

which is positive if $1<L e<1+\mu$ for any order-one value of $\tau>0$. Thus, for $\tau \neq \mathrm{o}(1)$, $\mu / \tau=\mathrm{O}(1)$ and $L e>1$, the flame ball is always unstable, having at least one positive root and possibly also a large positive root, regardless of the values of $a$ and $b$.

Only if $\tau /(1+\mu)=\mathrm{O}\left(\beta^{-2}\right)$ does the threshold for instability, with a large growth rate, rise above $L e=1$. We can see that, for $\tau /(1+\mu) \gg \beta^{-2}$ stability is only possible for $L e<1$, while for large values of $\mu$, with $\mu / \tau \gg \beta^{2}$, there are no large or order-one unstable eigenvalues for any order-one Lewis number. Because we have already covered the cases for large and order-one values of $\lambda$, the least stable eigenvalue would then have to be small as $\beta \rightarrow \infty$.

Small eigenvalues. When eigenvalues are small, an inspection of equation (40) reveals that $\lambda$ should be of the order of $\beta^{-2}$. The leading-order form of the dispersion relation, as $\beta \rightarrow \infty$, is then

$$
\left(\lambda+\frac{\mu \lambda}{1+\lambda \tau}+\frac{a^{2}}{\beta^{2}}\right)^{1 / 2}-(\lambda L e)^{1 / 2}=\frac{2-\frac{2}{3} b S^{2}}{\beta S}
$$

provided only that $(1+\mu) \lambda=\mathrm{o}(1)$. As will be seen, roots of the dispersion relation in the form (45) all satisfy $(1+\mu) \lambda=\mathrm{O}\left(\beta^{-2}\right)$, so that the analysis remains valid for any value of $\mu \geqslant 0$.

The case $a=0$. Considering firstly the situation in which $a=0$, it is convenient to define

$$
\lambda=\frac{\omega / \beta^{2}}{1+\mu} \quad \ell=\frac{L e}{1+\mu}
$$

which leads to the form of the dispersion relation

$$
\omega^{1 / 2}\left(1-\ell^{1 / 2}\right)=\frac{2-\frac{2}{3} b S^{2}}{S}
$$

to leading order as $\beta \rightarrow \infty$, provided only that $\tau \ll \beta^{2}$. The right-hand side of this dispersion relation changes sign where $b S^{2}=3$; this happens at the fold bifurcation in the curve for $a=0$ in figure 3 . On this curve, the right-hand side of equation (47) is positive $\left(b S^{2}<3\right)$ for 
$1 \leqslant S<\sqrt{\mathrm{e}}$, corresponding to the branch of smaller flame balls, and negative $\left(b S^{2}>3\right)$ for $S>\sqrt{\mathrm{e}}$, corresponding to larger flame balls.

It should be remembered that $\omega^{1 / 2}$ and $(\omega \ell)^{1 / 2}$, which represent scaled versions of $\kappa$ and $\nu$, must have non-negative real parts for solutions to arise. This means that an eigenvalue $\lambda$ of the order of $\beta^{-2}$ appears only if $b S^{2}<3$ with $\ell<1$ or $b S^{2}>3$ with $\ell>1$. The eigenvalue is real and positive, indicating that smaller flame balls $\left(b S^{2}<3\right)$ are unstable for $L e<1+\mu$ and larger flame balls $\left(b S^{2}>3\right)$ are unstable for $L e>1+\mu$. Since we have already seen that, at least for $\tau \gg \beta^{-2}$, stability is only possible for $L e<1$, it follows that, when $a=0$, the larger branch of flame balls is stable for $L e<1$.

The case $a \neq 0$. Now considering the more general situation in which $a \neq 0$, it is convenient to define

$$
\lambda=\Lambda \frac{a^{2} / \beta^{2}}{1+\mu} \quad \ell=\frac{L e}{1+\mu} \quad G=\frac{2-\frac{2}{3} b S^{2}}{a S}
$$

which leads to the form of the dispersion relation

$$
(1+\Lambda)^{1 / 2}-(\Lambda \ell)^{1 / 2}=G
$$

again provided only that $\tau \ll \beta^{2}$. Note that, as in the case $a=0$, the right-hand side $G$ of this dispersion relation changes sign where

$$
b S^{2}=3 \quad \text { or } \quad a S=\ln S^{2}-1
$$

both of which are plotted as the upper broken curves in figures 2 and 3 . The eigenvalues can be determined as solutions of the quadratic equation for $\Lambda^{1 / 2}$

$$
(1-\ell) \Lambda-2 \ell^{1 / 2} G \Lambda^{1 / 2}+1-G^{2}=0 \quad \text { or } \quad \Lambda^{1 / 2}=\frac{\ell^{1 / 2} G \pm \sqrt{G^{2}+\ell-1}}{1-\ell}
$$

requiring, at least, that $G \geqslant 0$ for $\ell<1$ so that $\Lambda^{1 / 2}$ has a non-negative real part. The eigenvalue $\Lambda$ itself has a negative real part (implying stability) when $\operatorname{Im}\left(\Lambda^{1 / 2}\right)>\operatorname{Re}\left(\Lambda^{1 / 2}\right)$.

For $L e \geqslant 1+\mu$ (heavy reactants), equivalent to $\ell \geqslant 1$. As has already been shown, there is a large positive eigenvalue for $L e>1+\mu$ so that this range of Lewis numbers is unstable regardless of the existence or not of any small eigenvalues. It is more relevant therefore to consider $\ell<1$. All the same, positive values of $\Lambda$ are found for all $G<1$, also making this region unstable to a disturbance with a weakly positive eigenvalue. If $G=1$ then $\Lambda=0$. For $G>1$ there are no solutions, real or complex, for which $(1+\Lambda)^{1 / 2}$ and $(\Lambda \ell)^{1 / 2}$ have a non-negative real part.

For $L e<1+\mu$ (light reactants or $\mu \gg 1$ ). For $\ell<1$, at least one positive real value of $\Lambda$ is found for all

$$
G \geqslant(1-\ell)^{1 / 2}
$$

two real values of $\Lambda \geqslant 0$ being found in the range

$$
(1-\ell)^{1 / 2}<G \leqslant 1 .
$$

In the range

$$
\left(\frac{1-\ell}{1+\ell}\right)^{1 / 2}<G<(1-\ell)^{1 / 2}
$$


complex conjugate pairs of values of $\Lambda$ are found with a positive real part, while smaller values of $G$ than the lower bound of this range produce non-positive real parts for $\Lambda$. Thus small eigenvalues become stable for

$$
G<\left(\frac{1-\ell}{1+\ell}\right)^{1 / 2}=\left(\frac{1+\mu-L e}{1+\mu+L e}\right)^{1 / 2} .
$$

The right-hand side of this inequality is in the range $[0,1)$ and increases towards unity as the solid heat capacity $\mu$ increases or as the Lewis number $L e$ decreases towards zero.
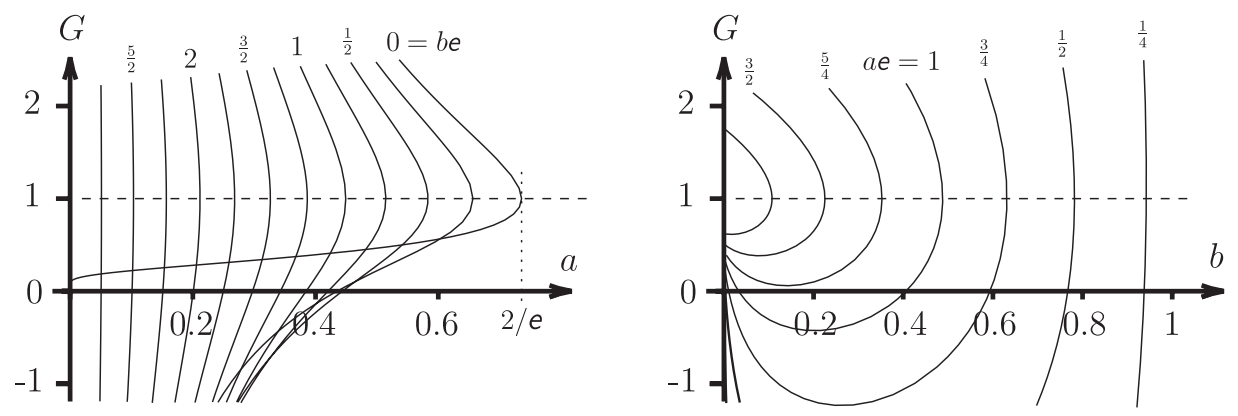

Figure 4. Variation of the instability parameter $G$ with $a$ at fixed values of $b$ (left) and with $b$ at fixed values of $a$ (right). The lower branches of values of $G$, at fixed $a$ and $b$, correspond to branches of solution with larger radii $S$ and larger values of $\beta(1-\widehat{T})$ in figures 2 and 3 .

Ranges of stability. To summarize, if $\beta^{-2} \ll \tau \ll \beta^{2}$ then stability arises for $a \neq 0$ when

$$
L e<1 \quad \text { and } \quad G<\left(\frac{1+\mu-L e}{1+\mu+L e}\right)^{1 / 2} .
$$

If $\tau=\mathrm{O}\left(\beta^{-2}\right)$ then stability arises for $a \neq 0$ when

$$
L e<L e_{\mathrm{c}}\left(\mu, \beta^{2} \tau, S\right) \quad \text { and } \quad G<\left(\frac{1+\mu-L e}{1+\mu+L e}\right)^{1 / 2}
$$

where $1 \leqslant L e_{\mathrm{c}}\left(\mu, \beta^{2} \tau, S\right) \leqslant 1+\mu$, with $L e_{\mathrm{c}}(\mu, 0, S)=1+\mu$ and $L e_{\mathrm{c}}(\mu, \infty, S)=1$. It is probably unrealistic to encounter such small heat transfer times in practice, so that the former pair of criteria above is likely to be the most practical set of conditions for $\mu=\mathrm{O}(1)$. When $\mu$ is large, and $\mu / \tau \gg \beta^{2}$, the value of $L e_{\mathrm{c}}$ becomes infinite so that only the condition $\mathrm{G}<1$ is needed to determine stability.

In order to determine where different solutions are stable or unstable, we must now relate the parameter $G$ to the values of $a, b$ and $S$. From (28) and (48) we have the pair of relations

$$
G=\frac{2-\frac{2}{3} b S^{2}}{a S} \quad \text { with } \quad a S=\ln S^{2}-\frac{1}{3} b S^{2}
$$

which can be used to parametrize the relationship between $G$ and $a$ at fixed values of $b$ or between $G$ and $b$ at fixed values of $a$. Both forms of variation are plotted in figure 4. In practice, we have seen that stability arises only for $G<G_{\mathrm{s}}=\left(\frac{1-\ell}{1+\ell}\right)^{1 / 2}<1$. Figure 4 shows that this tends to occur more readily towards smaller values of $a$ and larger values of $b$. The flame-ball solution on the branch having smaller values of $S$ is always unstable since it gives $G>1$ in all cases. 


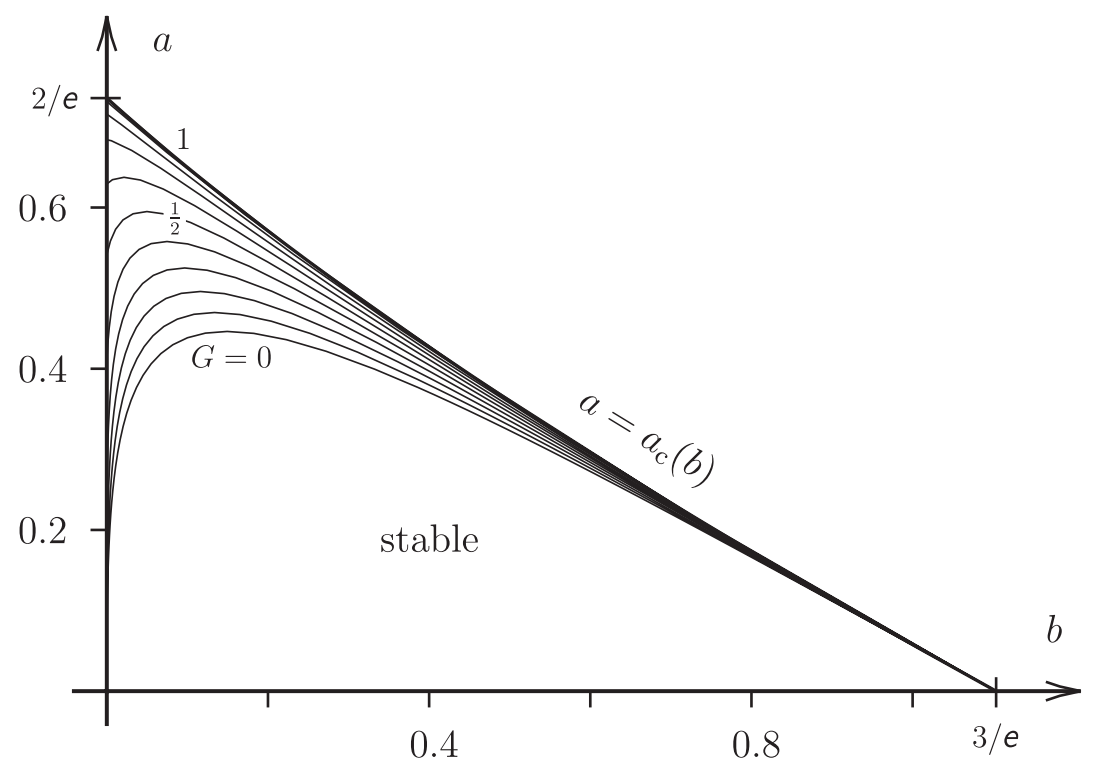

Figure 5. Contours of fixed $G$ between zero and unity in the space of heat loss parameters $(a, b)$. Solutions for $L e<1$ and $\mu \geqslant 0$ are stable to spherically symmetric disturbances only for $G<\left(\frac{1+\mu-L e}{1+\mu+L e}\right)^{1 / 2}$, which happens on the branch having larger values of $S$.

The formulae

$$
a=2 \frac{\ln S^{2}-1}{S(2-G)} \quad \text { and } \quad b=3 \frac{2-G \ln S^{2}}{S^{2}(2-G)}
$$

parametrize the relationship between $a$ and $b$ at fixed values of $G$. It can be noted that the case $G=1$ corresponds exactly to the limit of existence of solutions $a=a_{\mathrm{c}}(b)$ provided by equations (29). Based on these formulae, figure 5 provides the range of values of $a$ and $b$ for which stable solutions are possible when $L e<1$. Solutions for $L e<1$, on the branch having larger values of $S$, are stable when $G<G_{\mathrm{s}}$, with $0 \leqslant G_{\mathrm{s}} \leqslant 1$, so that the contour for $G=G_{\mathrm{s}}$ determines the limit of the range of values of $a$ and $b$ within which stable solutions can occur. To illustrate the dependence of stability on the steady radius $S$, the broken curves in figures 2 and 3 show the paths of the limiting cases at which $G=0$ and 1. Flame balls larger than the radius at which $G=0$ are always stable for any $L e<1$, while those that are smaller than the radius at which $G=1$ are always unstable.

Because the paths $G=1$ and $a=a_{\mathrm{c}}(b)$ are the same, based on the linearized model for heat loss in equations (18) that we are currently considering, the entire range of larger flame-ball solutions becomes stable for all possible values of $a \leqslant a_{\mathrm{c}}(b)$ only as $\mu \rightarrow \infty$ or $L e \rightarrow 0$, in which case $G_{\mathrm{s}} \rightarrow 1$. For $\mu<\infty$ and $L e>0$ a path of constant $G$ between $G=0$ and 1 divides stable from unstable flame balls.

In figure 6 we present the eigenvalues $\lambda$ that arise in a case for which $G=1 / \sqrt{3}$. The value of $\ell$ at which $\lambda$ has a real part of zero is then exactly $\frac{1}{2}$ with $\lambda$ becoming real at $\ell=\frac{2}{3}$. A numerical calculation on the larger branch of flame balls for $\beta=100$, with $b \mathrm{e}=1, a=0.48$ and $\mu=0$, gives almost exactly the same dependence of $\lambda$ on $\ell$, even though leading-order asymptotic solutions do not actually exist, as $\beta \rightarrow \infty$, at these values of $a$ and $b$; at this point, $a>a_{\mathrm{c}}(1 / \mathrm{e}) \approx 0.44914$. In order to find $G=1 / \sqrt{3}$ with $b \mathrm{e}=1$, a value of $a \approx 0.43619$ would be expected in the limit as $\beta \rightarrow \infty$. 


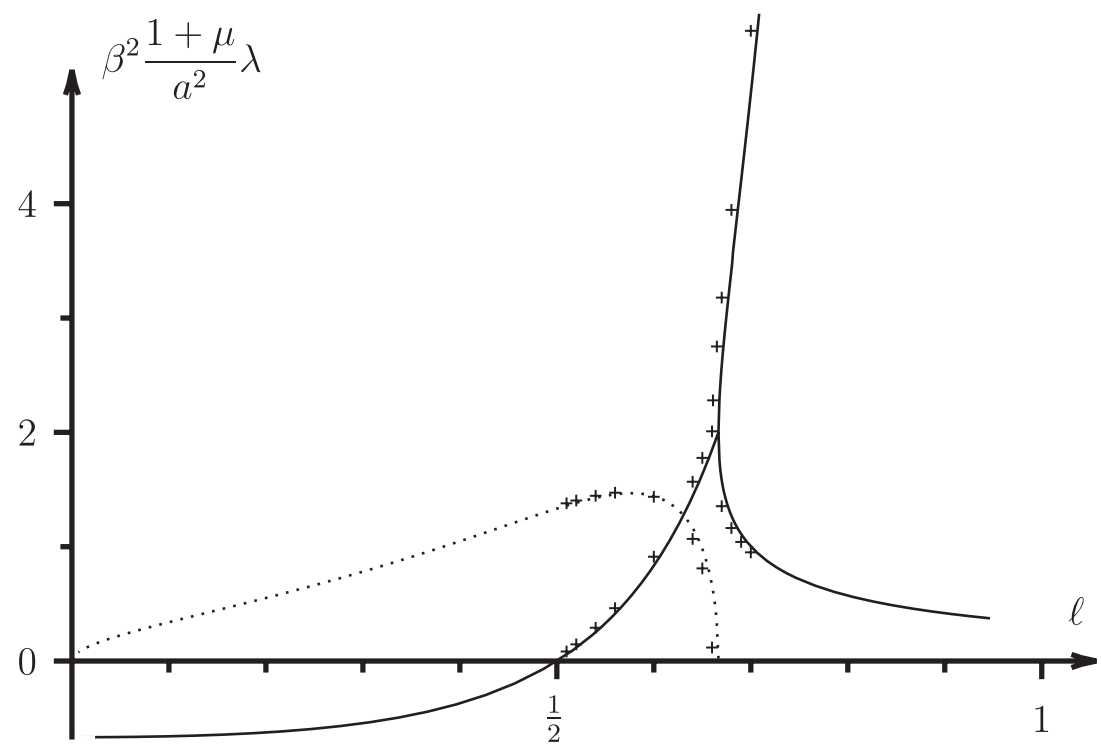

Figure 6. Real and imaginary parts of the eigenvalue $\lambda$ for a case in which $G=1 / \sqrt{3}$ (leading to stability when $\ell<\frac{1}{2}$ ). The imaginary part is given by the dotted curve. The small + signs denote numerically calculated eigenvalues for $\beta=100$ with $b$ e $=1, a=0.48$ and $\mu=0$, which happens to have almost the same point for marginal stability.

Nevertheless, the dependence of the numerical eigenvalues on $\ell$ is remarkably well predicted. The difference in the values of $a$ and $b$ used in these numerical calculations from values that would give $G=1 / \sqrt{3}$ as $\beta \rightarrow \infty$ is entirely consistent with the differences, of the order of $10 \%$, seen in figures 2 and 3. This diagram demonstrates that the stability characteristics of the numerical solutions that are found for $\beta=100$ (and by extension any other large enough value of $\beta$ ) at given values of $a$ and $b$ are remarkably well predicted by the dispersion relation (49) for a value of $G$ that is found at some nearby values of $a$ and $b$.

\subsection{Stefan's law for heat loss}

3.2.1. Steady solutions. When the equation involving nonlinear radiation, distributed over all values of $r$, is satisfied, namely

$$
T_{t}+\mu \theta_{t}=T_{r r}+\frac{2}{r} T_{r}-\frac{b}{\beta} T^{4}-\frac{a^{2}}{\beta^{2}} T
$$

a steady asymptotic solution for the solid and gas temperatures as $\beta \rightarrow \infty$ becomes

$\theta=T=T_{0}(r)$

$$
\sim \begin{cases}\widehat{T} \frac{S}{r} \frac{\sinh (a r / \beta)}{\sinh (a S / \beta)}+\left(\frac{b}{\beta} \widehat{T}^{4}-\frac{2 b^{2}}{3 \beta^{2}} \widehat{T}^{7} S^{2}\right) \frac{r^{2}-S^{2}}{6}+\frac{b^{2}}{\beta^{2}} \widehat{T}^{7} \frac{r^{4}-S^{4}}{30} & \text { if } \quad r<S \\ \widehat{T} \frac{S}{r} \mathrm{e}^{a(S-r) / \beta}+\frac{b}{2 \beta} \widehat{T}^{4}\left(\frac{S^{4}}{r^{2}}-S^{2}\right) \mathrm{e}^{4 a(S-r) / \beta} & \text { if } \quad r>S .\end{cases}
$$

This solution is exact if $b=0$, and can be arrived at by iteration starting from the solution for $b=0$. As written here, errors are of the order of $\left(b^{3}+a^{2} b\right) \beta^{-3}$ for $r<S$ and, at most, of the order of $\left(b^{2}+a b\right) T_{0} \beta^{-2}$, for all values of $r>S$. 
The jump conditions $\left[Y_{r}\right]=-\left[T_{r}\right]=\mathrm{e}^{\beta(\widehat{T}-1) / 2}$, evaluated to order $\beta^{-1}$, then yield

$$
\beta(1-\widehat{T})=\ln S^{2}=a S+\frac{4}{3} b S^{2}+\mathrm{O}\left(\beta^{-1}\right)
$$

which is completely analogous to the previous result (28) for constant heat losses in the burnt gas and linear losses in the unburnt gas. If the earlier parameter $b$ is replaced by $4 b$, for values of $a$ and $b$ in the appropriate range $a \leqslant a_{\mathrm{c}}(4 b)$, then it provides the same range of steady solutions, with asymptotically the same values of $S$ and $\widehat{T}$. In the case when $b=0$ the solutions are also asymptotically the same as those of the model (18) with $b=0$, in spite of the fact that linear heat losses are included in the model (60) for $r<S$. In this region, these linear losses produce a gradient $T_{r}$ for $r<S$ that is only of the order of $\beta^{-2}$. They therefore contribute nothing to the solution to leading order.

3.2.2. Spherically symmetric stability. In proceeding to consider the linear stability of the solution (61) we suppose, as before, that $T=T_{0}+\mathrm{e}^{\lambda t} \Gamma(r)$. The linearized equation to be satisfied by $\Gamma(r)=(1+\lambda \tau) \Theta(r)$ can then be taken as

$$
\begin{aligned}
\Gamma^{\prime \prime}+\frac{2}{r} \Gamma^{\prime}=( & \\
& \left.+\frac{\mu d}{1+\lambda \tau}+\frac{a^{2}}{\beta^{2}}\right) \Gamma \\
& \text { for } r<S
\end{aligned}
$$

As before, the solution for $y(r)$ is

$$
y= \begin{cases}0 & \text { if } \quad r<S \\ -\frac{1}{r} \mathrm{e}^{v(S-r)} & \text { if } \quad r>S\end{cases}
$$

and, because heat losses are continuous across $r=S$ for the model (60), we now have

$$
-\left[T_{0}^{\prime}\right]=1 / S \quad\left[T_{0}^{\prime \prime}\right]=2 / S^{2} .
$$

Hence the jump conditions, equivalent to (32), to be satisfied by $\Gamma$ become

$$
[\Gamma]=1 / S \quad-\left[\Gamma^{\prime}\right]=\frac{1}{S^{2}}+\frac{v}{S}=\frac{2}{S^{2}}+\frac{b}{6}+\frac{\beta}{2 S} \Gamma\left(S^{-}\right)+\mathrm{O}\left(\beta^{-1}\right)
$$

since $y^{\prime}\left(S^{+}\right)=S^{-2}+v / S$.

It remains to solve for $\Gamma$ and to apply these jump conditions. The solution for $r<S$ is straightforward, namely

$\Gamma=\widehat{\Gamma}_{\mathrm{b}} \frac{S}{r} \frac{\sinh (\delta r)}{\sinh (\delta S)} \quad$ with $\quad \delta^{2}=\lambda+\frac{\lambda \mu}{1+\lambda \tau}+\frac{4 b}{\beta}\left(1-\frac{\ln S^{6}}{\beta}\right)+\frac{a^{2}}{\beta^{2}}$.

Note that we have redefined the value of $\delta$ from (35) although the parameter still applies to the solution for $\Gamma$ where $r<S$. The solution for $r>S$ is less straightforward in view of the non-constant coefficient. We need to solve

$\Gamma^{\prime \prime}+\frac{2}{r} \Gamma^{\prime}=\left(\frac{\varpi^{2}}{\beta} \frac{\mathrm{e}^{-3 a r / \beta}}{r^{3}}+\kappa^{2}\right) \Gamma \quad$ with $\quad \varpi^{2}=4 b S^{3}\left(1-\frac{\ln S^{6}}{\beta}\right) \mathrm{e}^{3 a S / \beta}$

where $\kappa$ is defined in (35). This equation appears to have no standard solution for general values of $\kappa, \varpi$ and $a / \beta$. However, we can still proceed in the following way. 
Recalling that the large and order-one unstable eigenvalues, for $L e>1$, that arose in the previous model with constant heat loss in the burnt gas, did not depend on the heat loss parameters, we can expect the same to be true in this case. Indeed, when $\lambda \geqslant \mathrm{O}(1)$ the terms containing $a$ and $b$ are negligible in the differential equation (63) for the eigenfunction $\Gamma(r)$. Exactly the same eigenvalues (to leading order as $\beta \rightarrow \infty$ ) should therefore arise in this case. There is no real need to repeat the analysis for this.

On the other hand, we can also anticipate that small eigenvalues, relevant only for $L e<1$, will be of the order of $\beta^{-2}$ as before. We then find

$$
\Gamma^{\prime \prime}+\frac{2}{r} \Gamma^{\prime}=\left(\frac{\varpi^{2}}{\beta} \frac{1}{r^{3}}-\frac{\varpi^{2} a}{\beta^{2}} \frac{3}{r^{2}}+\frac{\chi^{2}}{\beta^{2}}\right) \Gamma \quad \text { where } \quad \kappa^{2}=\frac{\chi^{2}}{\beta^{2}}
$$

to order $\beta^{-2}$ for $r=\mathrm{O}(1)$, provided $\tau \ll \beta^{2}$, as well as the form of the equation, rewritten for large values of $r$

$$
\frac{\mathrm{d}^{2} \Gamma}{\mathrm{d} z^{2}}+\frac{2}{z} \frac{\mathrm{d} \Gamma}{\mathrm{d} z}=\left(\chi^{2}+\frac{\varpi^{2}}{\beta^{2}} \frac{\mathrm{e}^{-3 a z}}{z^{3}}\right) \Gamma \quad \text { with } \quad r=\beta z
$$

for $z=\mathrm{O}(1)$, also to order $\beta^{-2}$. Asymptotic solutions of each of these equations as $\beta \rightarrow \infty$ are, respectively,

$$
\begin{aligned}
\Gamma \sim A\left(\frac{1}{r}+\frac{\varpi^{2}}{\beta}\right. & \left.\frac{1}{2 r^{2}}+\frac{\varpi^{2} a}{\beta^{2}} 3 \frac{\ln r+1}{r}+\frac{\varpi^{4}}{\beta^{2}} \frac{1}{12 r^{3}}+\frac{\chi^{2}}{\beta^{2}} \frac{r}{2}\right) \\
+ & B\left(1-\frac{\varpi^{2}}{\beta} \frac{\ln r+1}{r}-\frac{\varpi^{2} a}{\beta^{2}}\left(\ln r^{3}+3\right)-\frac{\varpi^{4}}{\beta^{2}} \frac{\ln r+\frac{5}{2}}{2 r^{2}}+\frac{\chi^{2}}{\beta^{2}} \frac{r^{2}}{6}\right)
\end{aligned}
$$

with errors that are of the order of $A \beta^{-3}$ or $B \beta^{-3}$ for $r=\mathrm{O}(1)$, and, after noting that $\Gamma$ must be bounded as $z \rightarrow \infty$

$$
\begin{aligned}
\Gamma \sim C \frac{\mathrm{e}^{-\chi z}}{z}+ & C \frac{\varpi^{2}}{\beta^{2}}\left(\frac{\mathrm{e}^{-(\chi+3 a) z}}{2 z^{2}}+\frac{9 a^{2}}{4 \chi} \frac{\mathrm{e}^{-\chi z} \operatorname{Ei}_{1}(3 a z)}{z}\right. \\
& \left.-\left(\chi+3 a+\frac{9 a^{2}}{4 \chi}\right) \frac{\mathrm{e}^{\chi z} \operatorname{Ei}_{1}((2 \chi+3 a) z)}{z}\right) \\
= & C\left(\frac{1}{z}-\chi+\frac{\varpi^{2}}{\beta^{2}} \frac{1}{2 z^{2}}+\frac{\varpi^{2}}{\beta^{2}}(\chi+3 a) \frac{\ln z}{z}+\mathrm{O}\left(z, \frac{\beta^{-2}}{z}\right)\right) \quad \text { as } \quad z \rightarrow 0
\end{aligned}
$$

involving errors that are of the order of $C \beta^{-4}$ for $z=r / \beta=\mathrm{O}(1)$. In this result, the function $\operatorname{Ei}_{1}(z)=\int_{1}^{\infty}\left(\mathrm{e}^{-z t} / t\right) \mathrm{d} t=-\ln z-\gamma+z+\mathrm{O}\left(z^{2}\right)$ is the first exponential integral function and $\gamma$ is Euler's constant. Written in terms of $r$, which is taken to be such that $1 \ll r \ll \beta$, the latter solution gives

$$
\Gamma=C\left(\beta\left(\frac{1}{r}+\frac{\varpi^{2}}{\beta} \frac{1}{2 r^{2}}\right)-\chi+\mathrm{O}\left(\frac{r}{\beta}, \frac{\ln \beta}{\beta r}\right)\right)
$$

which matches the solution (71) to the order of terms neglected only if

$$
C=\beta^{-1} A+\mathrm{O}\left(\frac{\ln \beta}{\beta^{2}}\right) \quad \text { and } \quad B=-\beta^{-1} \chi A+\mathrm{O}\left(\frac{\ln \beta}{\beta^{2}}\right) .
$$

The asymptotic solution for order-one values of $r>S$ is therefore

$$
\Gamma \sim A\left(\frac{1}{r}+\frac{\varpi^{2}}{\beta} \frac{1}{2 r^{2}}-\frac{\chi}{\beta}\right)
$$




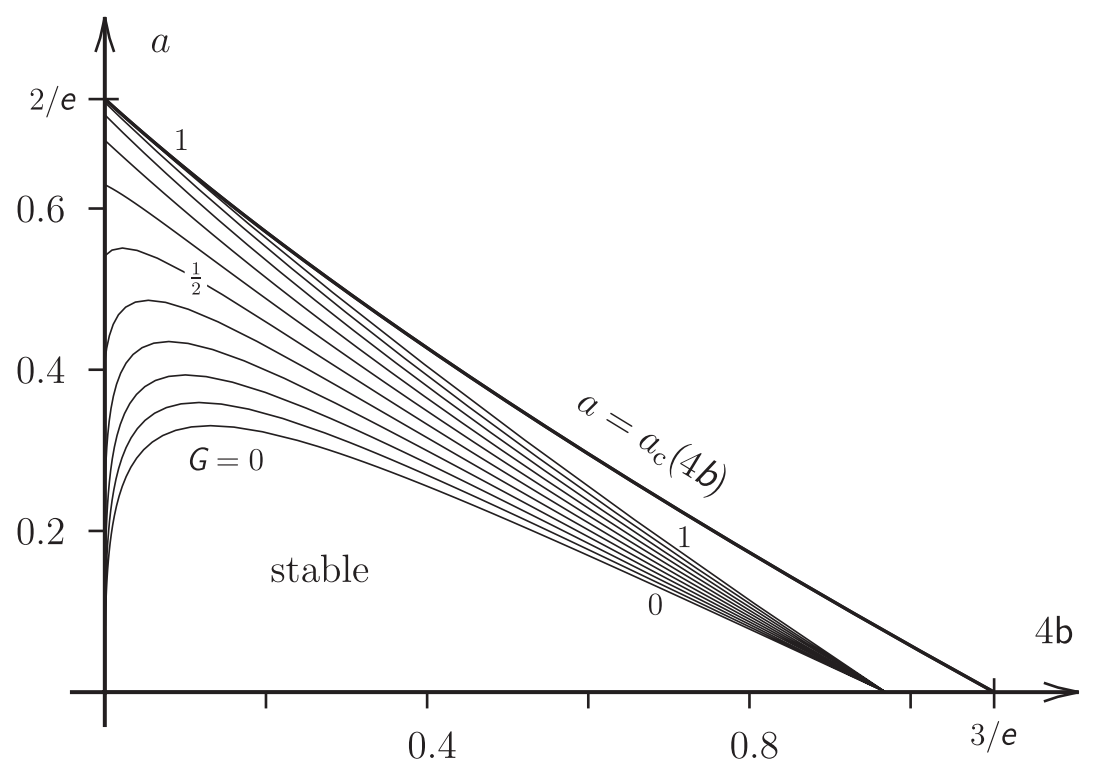

Figure 7. Contours of fixed $G$ between zero and unity in the space of heat loss parameters $(a, b)$ Solutions with distributed radiative losses for $L e<1$ and $\mu \geqslant 0$ are stable to spherically symmetric disturbances only for $G<\left(\frac{1+\mu-L e}{1+\mu+L e}\right)^{1 / 2}$.

with errors of the order of $A \beta^{-2} \ln \beta$ as $\beta \rightarrow \infty$.

The jump conditions (66) can now be used to deduce that

$$
\begin{aligned}
& A\left(1-\kappa S+2 \frac{b}{\beta} S^{2}\right)-\widehat{\Gamma}_{\mathrm{b}} S=1 \\
& A\left(1+4 \frac{b}{\beta} S^{2}\right)+\widehat{\Gamma}_{\mathrm{b}}\left(\delta S^{2} \operatorname{coth}(\delta S)-S\right)=1+\nu S=2+\frac{1}{6} b S^{2}+\frac{1}{2} \beta S \widehat{\Gamma}_{\mathrm{b}}
\end{aligned}
$$

after using (68) and (69) to substitute for $\varpi$ and $\chi$, and neglecting terms of the order of $\beta^{-2} \ln \beta$ or less. It can thus be seen that

$$
\widehat{\Gamma}_{\mathrm{b}}=\frac{v-\kappa-2 \frac{b}{\beta} S-\kappa \nu S+2 \frac{b}{\beta} \nu S^{2}}{\left(1-\kappa S+2 \frac{b}{\beta} S^{2}\right) \delta S \operatorname{coth}(\delta S)+2 \frac{b}{\beta} S^{2}+\kappa S}=\frac{2 v S-2-\frac{1}{3} b S^{2}}{\beta S}
$$

leading to the dispersion relation, for $\lambda=\mathrm{O}\left(\beta^{-2}\right)$

$$
\left(\lambda+\frac{\mu \lambda}{1+\lambda \tau}+\frac{a^{2}}{\beta^{2}}\right)^{1 / 2}-(\lambda L e)^{1 / 2}=\frac{2-\frac{5}{3} b S^{2}}{\beta S}
$$

after neglecting terms that are of the order of $\beta^{-2} \ln \beta$, remembering that for the small eigenvalues that we are examining (with $L e<1$ ) the values of $v$ and $\kappa$ are themselves of the order of $\beta^{-1}$. From (67) it can be seen that $\delta$ is of the order of $\beta^{-1 / 2}$ but its influence on the dispersion relation is still only to provide a disturbance that is of the order of $\delta^{2} / \beta=\mathrm{O}\left(\beta^{-2}\right)$. 
Ranges of stability. After comparing equations (45) and (78), it can be seen that stability arguments follow in the same way as they did for the model (17) and (18) with $G$ now defined (in place of the previous function $G$; note the change in font) as

$$
G=\frac{2-\frac{5}{12} b S^{2}}{a S} \quad \text { with } \quad \ln S^{2}=a S+\frac{1}{3} b S^{2} \quad \text { writing } b=4 b
$$

in which we have used the symbol $b$ instead of $4 b$ so that the analogy with (and difference from) the previous results can be seen more clearly. Flame balls are now found to be stable for $L e<1$ with $G<G_{\mathrm{s}}=\left(\frac{1+\mu-L e}{1+\mu+L e}\right)^{1 / 2}$. It can immediately be noted that $G>G$ for equivalent flame-ball solutions, except when $b=b=0$. It follows that flame balls are found to have a smaller range of stability when a more distributed nonlinear model for radiative heat loss is applied. Indeed, figure 7 shows the range of values of $a$ and $b$ (or $4 b$ ) over which $G$ varies between zero and unity. No flame balls are stable for $G>1$ and it can be seen that the boundary where $G=1$ is now distinct from the path of fold bifurcations at $a=a_{\mathrm{c}}(4 b)$, except where $b=0$. The paths at which $G=0$ and 1 are shown as dotted curves in figures 2 and 3. Except at $b=b=0$, these curves are found at larger steady radii $S$ than the corresponding broken curves where $G=0$ and 1 .

Broadly speaking therefore, the model with nonlinear distributed radiative losses moves the point at which flame balls become stable towards larger values of the steady radius $S$, above the path of fold bifurcations that separates larger from smaller solutions.

\section{Conclusions}

For a model describing flame balls in a porous solid, in which the solid serves only as a reservoir of heat and contributes nothing to conductive or radiative effects, the steady solutions themselves are exactly the same as they would be if no solid were present. The solid simply maintains the temperature that the gas would have in its absence. Although it certainly absorbs heat from the gas in reaching this state, it simply copies the gas temperature and does not influence it in any way. Neither does this removal of heat from the gas cause a reduction in gas temperature because the system is unbounded with an infinite reservoir of available enthalpy. However, in unsteady solutions, the reservoir of heat absorbed by the solid does make a difference, tending to stabilize steady flame balls, especially for Lewis numbers less than unity.

The principal results of our asymptotic analysis of flame balls as $\beta \rightarrow \infty$, in the presence of an inert solid matrix, are maintained over a very wide range of values of the heat transfer time $\tau$, between gas and solid. Provided only that $\beta^{-2} \ll \tau \ll \beta^{2}$ and $\mu=\mathrm{o}\left(\beta^{2} \tau\right)$, we find that all flame balls are unstable for Lewis numbers $L e$ greater than one. For $L e<1$ the presence of the solid increases the range of stable flame balls exactly as if the Lewis number $L e$ had been reduced to $L e /(1+\mu)$, at least to leading order as $\beta \rightarrow \infty$. If $\mu$ increases to the extent that $\mu \gg \beta^{2} \tau$, then the system behaves just as if the Lewis number was reduced to zero. All stable flame balls arise on the branch of solutions that has larger radii than those found below the path of fold bifurcations.

The nonlinear radiative law is found to produce exactly analogous steady solutions (to leading order as $\beta \rightarrow \infty$ ) to those found with the linearized law, related only by having $b=4 b$. Their stability is altered, however, in a way that narrows the range in which stable flame balls can be found. The stabilizing effect of heat exchange with the solid matrix is still present and serves to alter the effective Lewis number for stability, when $L e<1$, in exactly the same way. In both cases, lean reactants that are more diffusive than heat, can therefore 
produce flame balls that are stabilized more readily (with lower diffusivities) because of the exchange of heat with a solid matrix.

It can be recalled from equations (10) and (11) that $L e /(1+\mu)$ would serve as the Lewis number, in place of $L e$ in a model without the presence of any solid, if the heat transfer time $\tau$ were to be exactly zero. The eigenvalues that are found in the linear stability analysis when $L e<1$, are all small, of the order of $\beta^{-2}$. Thus any transfer time of $\tau \ll \beta^{2}$ is asymptotically small on the long time-scale of slow growth (or decay) of any eigenmode. It is therefore effectively zero, providing a ready explanation for the stabilizing effect that has been demonstrated.

On the other hand, for Lewis numbers that are greater than unity, $L e>1$, the eigenvalues are, in fact, typically very large, of the order of $\beta^{2}$. The time-scale for growth of the most unstable eigenmode is therefore very short, of the order of $\beta^{-2}$ and $\tau$ would then have to be very small indeed, of the order of $\beta^{-2}$, to have any effect on the stability of a flame ball. If, however, $\tau$ is very small, having $\tau \ll \beta^{-2}$, then the same stabilizing effect is found, effectively replacing the Lewis number in all cases by $L e /(1+\mu)$. Otherwise, if $\tau$ is less small, $\tau \gg \beta^{-2}$, then the solid simply has no influence at all (to leading order) on the instability of flame balls when $L e>1$. Thus, as far as the stability of a steady flame ball is concerned, intermediate heat transfer times, $\beta^{-2} \ll \tau \ll \beta^{2}$, behave as if $\tau$ was infinite for $L e>1$ and zero for $L e<1$.

The results hold for any value of $\mu \geqslant 0$, including relatively large values, representing situations in which the solid matrix takes up a larger volumetric heat capacity (or mass) than the gas phase, while still having a small-volume fraction. Situations in which the solid phase conducts and radiates, or possibly also moves itself, as may happen in the case of a dust suspension, remain to be studied. This paper has focused only on the primary role of the transfer of heat between the solid and gas.

Numerical results are found to require unusually large activation temperatures in order to approach the asymptotic results. Values of $\beta$ of about 20 are found to produce solutions that are different both qualitatively and quantitatively from the asymptotic solutions for $\beta=\infty$. We believe that our numerical solutions are reliable: at $\beta=100$ the steady solutions are consistent with the asymptotic results; the dependence of numerically calculated unstable eigenvalues on the Lewis number is very close to that found asymptotically at nearby values of the heat loss parameters $a$ and $b$. Indeed, the numerical and asymptotic results support each other when $\beta$ is very large indeed (at least where our numerical investigations were carried out, with the linearized model for heat loss). It may be possible, but seems unlikely without any clear reason for it, that the numerics and asymptotics will agree better at smaller values of $\beta$ for the continuous, nonlinear radiative model. This remains to be investigated.

When $\beta$ is only moderately large, the numerical results are surprisingly different from their asymptotic counterparts. This raises some basic questions concerning the relevance of the asymptotic predictions for one-step chemistry in describing actual flame balls, questions that deserve further investigation; numerical simulations based on more detailed hydrogen chemistry appear to be successful [7-19]. Apart from this caveat, the analysis in this paper has served to clarify and extend what is known about flame balls, as seen from the asymptotic perspective. Studies of this nature [8-12] have proven successful at describing experimental observations of flame balls, and their behaviour, with good qualitative accuracy, at low enough Lewis numbers.

\section{Acknowledgments}

The authors are grateful to the EPSRC for financial support and to the IMA in Minneapolis for academic and computing support as well as hospitality. 


\section{References}

[1] Zeldovich Ya B, Barrenblatt G I, Librovich V B and Makhviladze G M 1985 The Mathematical Theory of Combustion and Explosions (New York: Consultants Bureau)

[2] Deshaies B and Joulin G 1984 On the initiation of a spherical flame kernel Combust. Sci. Technol. 37 99-116

[3] Joulin G 1985 Point source initiation of lean spherical flames of light reactants: an asymptotic study Combust. Sci. Technol. 4399

[4] Champion M, Deshaies B, Joulin G and Kinoshita K 1986 Spherical flame initiation: theory vs experiments for lean $\mathrm{C}_{3} \mathrm{H}_{8}$-air mixtures Combust. Flame 65 319-37

[5] Ronney P D, Wu M S, Pearlman H G and Weiland K J 1998 Experimental study of flame balls in space: Preliminary results from STS-83 AIAA J. 36 1361-8

[6] Buckmaster J D, Joulin G and Ronney P 1990 The structure and stability of nonadiabatic flame balls Combust. Flame 79 381-92

[7] Buckmaster J D, Joulin G and Ronney P 1991 The structure and stability of nonadiabatic flame balls: the effects of far-field losses Combust. Flame 84 411-22

[8] Buckmaster J and Joulin G 1991 Flame balls stabilized by suspension in fluid with a steady linear ambient velocity distribution J. Fluid Mech. 227 407-27

[9] Buckmaster J D and Joulin G 1993 Influence of boundary-induced losses on the structure and dynamics of flame-balls Combust. Sci. Technol. 89 57-69

[10] Ronney P D, Whaling K N, Abbudmadrid A, Gatto J L and Pisowicz V L 1994 Stationary premixed flames in spherical and cylindrical geometries AIAA J. 32 569-77

[11] Lozinski D, Buckmaster J and Ronney P 1994 Absolute flammability limits and flame-balls Combust. Flame 97 301-16

[12] Brailovsky I and Sivashinsky G I 1997 On stationary and travelling flame balls Combust. Flame 110 524-9

[13] Buckmaster J and Ronney P 1998 Flame ball drift in the presence of a total diffusive heat flux 27th Int. Symp. on Combustion (Pittsburgh, PA: Combustion Institute) pp 2603-10

[14] Joulin G, Kurdyumov V N and Liñán A 1999 Existence conditions and drift velocities of adiabatic flame-balls in weak gravity fields Combust. Theory Modelling 3 281-96

[15] Vederajan T G, Buckmaster J and Ronney P 1998 Two-dimensional failure waves and ignition fronts in premixed combustion 27th Int. Symp. on Combustion (Pittsburgh, PA: Combustion Institute) pp 537-44

[16] Lee C J and Buckmaster J 1991 The structure and stability of flame balls-a near-equidiffusional flame analysis SIAM J. Appl. Math. 51 1315-26

[17] Williams F A 1985 Combustion Theory (Menlo Park, CA: Benjamin-Cummings)

[18] Dold J W 2000 Reaction-sheet jump conditions in premixed flames Institute for Mathematics and its Applications (IMA) Preprint 1732 University of Minnesota, Minneapolis, USA

[19] Buckmaster J, Smooke M and Giovangigli V 1993 Analytical and numerical modeling of flame-balls in hydrogen-air mixtures Combust. Flame 94 113-24

[20] Abid M, Wu M S, Liu J B, Ronney P D, Ueki M, Maruta K, Kobayashi H, Niioka T and Vanzandt D M 1999 Experimental and numerical study of flame ball IR and UV emissions Combust. Flame 116 348-59

[21] Wu M S, Ronney P D, Colantonio R O and Vanzandt D M 1999 Detailed numerical simulation of flame ball structure and dynamics Combust. Flame 116 387-97 